\section{All-trans retinoic acid protects mesenchymal stem cells from immune thrombocytopenia by regulating the complement-interleukin-1 $\beta$ loop}

\author{
Xiaolu Zhu, ${ }^{1}$ Yanan Wang, ${ }^{1}$ Qian Jiang, ${ }^{1}$ Hao Jiang, ${ }^{1}$ Jin Lu, ${ }^{1}$ Yazhe Wang, ${ }^{1}$ \\ Yuan Kong, ${ }^{1}$ Yingjun Chang, ${ }^{1}$ Lanping $\mathrm{Xu},{ }^{1}$ Jun Peng, ${ }^{2}$ Ming Hou, ${ }^{2}$ Xiaojun \\ Huang $^{1}$ and Xiaohui Zhang ${ }^{1}$
}

${ }^{1}$ Peking University People's Hospital, Peking University Institute of Hematology, Beijing Key Laboratory of Hematopoietic Stem Cell Transplantation, National Clinical Research Center for Hematologic Disease, Beijing and ${ }^{2}$ Department of Hematology, Qilu Hospital, Shandong University, Jinan, P.R. China

\section{ABSTRACT}

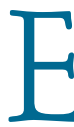
nhanced peripheral complement activation has long been considered as one of the major pathogenic elements of immune thrombocytopenia. A dysfunctional bone marrow microenvironment, especially with regards to mesenchymal stem cells, has been observed in patients with immune thrombocytopenia. However, the potential role of the complement system in the dysfunctional bone marrow microenvironment remains poorly understood. In this study, bone marrow samples from patients with immune thrombocytopenia were divided into two groups based on whether or not complement components were deposited on the surfaces of their mesenchymal stem cells. The mesenchymal cells from the group with complement deposition were less numerous, dysfunctional, had a reduced capacity to proliferate, and showed increased apoptosis as well as abnormal secretion of interleukin-1 $\beta$ and $\mathrm{C}-\mathrm{X}-\mathrm{C}$ motif chemokine ligand 12 . In vitro treatment with all-trans retinoic acid increased the number and improved the function of the complement-positive bone marrow mesenchymal stem cells by upregulating DNA hypermethylation of the interleukin-1 $\beta$ promoter. In vivo studies showed that all-trans retinoic acid could rescue the impaired mesenchymal stem cells to support the thrombopoietic niche in both patients with immune thrombocytopenia and a murine model of this disease. Taken together, these results indicate that impairment of mesenchymal stem cells, mediated by the complement-interleukin-1 $\beta$ loop, plays a role in the pathogenesis of immune thrombocytopenia. All-trans retinoic acid represents a promising therapeutic approach in patients with immune thrombocytopenia through its effect of repairing mesenchymal stem cell impairment.

\section{Introduction}

Immune thrombocytopenia (ITP) is a common autoimmune disorder characterized by severe isolated thrombocytopenia. ${ }^{1}$ Increasing evidence suggests a role for complement activation in ITP. ${ }^{1-3}$ We previously characterized the abnormal enhanced complement activation in plasma samples from patients with ITP, as well as enhanced plasma complement activation/fixation capacity on immobilized heterologous platelets. ${ }^{4}$ Moreover, we confirmed the activation of both the classical and alternative complement pathways in the peripheral blood of patients with ITP. ${ }^{4}$ However, our knowledge regarding the involvement of the complement system in the bone marrow of patients with ITP is still very limited.

Emerging evidence indicates that complement affects not only B-cell responses ${ }^{5,6}$ but also T-cell immunity during the induction, effector and contraction phases of an immune response. ${ }^{7-10}$ Interestingly, we and others have identified an imbalance between B-effector and T-regulatory networks involved in the pathogenesis of ITP. ${ }^{1,11-14}$ Mesenchymal stem cells (MSC) have been documented to play crucial
Ferrata Storti Foundation

Haematologica 2019

Volume 104(8):1661-1675

Partial results of this research were presented as an oral presentation at the $58^{\text {th }}$ American

Society of Hematology Annual Meeting and Exposition (ASH), San Diego, CA, 3 December 2016 ("Crucial Role of Complement Activation and IL-1 $\beta$ in Bone Marrow Niche of Immune Thrombocytopenia", n. 166).

\section{Correspondence:}

XIAOHUI ZHANG

zhangxh100@sina.com

Received: August 13, 2018.

Accepted: January 21, 2019.

Pre-published: January 24, 2019.

doi:10.3324/haematol.2018.204446

Check the online version for the most updated information on this article, online supplements, and information on authorship \& disclosures: www.haematologica.org/content/104/8/1661

\section{(C)2019 Ferrata Storti Foundation}

Material published in Haematologica is covered by copyright. All rights are reserved to the Ferrata Storti Foundation. Use of published material is allowed under the following terms and conditions:

https://creativecommons.org/licenses/by-nc/4.0/legalcode. Copies of published material are allowed for personal or internal use. Sharing published material for non-commercial purposes is subject to the following conditions: https://creativecommons.org/licenses/by-nc/4.0/legalcode, sect. 3. Reproducing and sharing published material for commercial purposes is not allowed without permission in writing from the publisher. 
roles in immune modulatory functions with effects on $\mathrm{T}$ and B-cell activation. ${ }^{15,16}$ Notably, we found that MSC from ITP patients exhibited increased apoptosis and senescence, which was associated with the regulation of T-cell subsets. ${ }^{17-19}$ However, the underlying mechanisms of the dysfunction of MSC in ITP bone marrow remain unclear. We, therefore, wondered whether complement activation in bone marrow was associated with defective MSC in ITP.

Complement components can enhance pro-inflammatory receptor-mediated signaling in phagocytes, leading to increased production of interleukin-1 $\beta$ (IL-1 $\beta$ ). ${ }^{20-22} \mathrm{IL}-1 \beta$ is critically involved in several inflammatory diseases and its levels have been reported to be elevated in ITP. ${ }^{23}$ Interestingly, bone marrow MSC have been demonstrated to be capable of synthesizing and releasing IL-1 $\beta .^{24,25}$

All-trans retinoic acid (ATRA) has revolutionized the therapy of acute promyelocytic leukemia. ${ }^{26}$ We previously reported that the combination of ATRA with any one of methylprednisolone, danazol or cyclosporine A produced better responses in patients with corticosteroid-resistant or relapsed ITP $\left(54^{\text {th }}\right.$ American Society of Hematology Annual Meeting and Exposition; Poster ID: 3338). Recently we also reported the findings of a multicenter, randomized, open-label, phase II trial, suggesting that ATRA represents a promising candidate treatment for patients with corticosteroid-resistant or relapsed ITP. ${ }^{27}$ Panzer and Pabinger positively appraised our findings of a high response rate to ATRA as well as the few, mild adverse events associated with this drug compared with other second-line treatments for ITP. ${ }^{28}$ However, few studies have focused on the mechanisms underlying the effects of ATRA..$^{29}$ Furthermore, the role of ATRA in regulating MSC function in ITP bone marrow is poorly understood. It has not been elucidated whether the complement system and associated pro-inflammatory cytokines are targeted by ATRA.

Here, we present evidence strongly suggesting that the complement-IL-1 $\beta$ loop mediates bone marrow MSC impairment in ITP. More importantly, ATRA protects MSC from dysfunction and apoptosis by upregulating DNA hypermethylation of the IL- $1 \beta$ promoter, which is conducive to restoring the thrombopoietic niche. We believe that these findings will serve to shift the focus of future studies on the complement system in the pathogenesis of ITP and interventions with ATRA to factors that regulate thrombocytopoiesis.

\section{Methods}

\section{Patients and study design}

The blood samples utilized in this study were collected between December 2016 and November 2017 from 58 consecutive, newly diagnosed ITP patients at the Institute of Hematology, Peking University People's Hospital, Beijing, China. Approval to take blood and bone marrow samples from healthy volunteers and patients was granted by the Ethics Committee of Peking University People's Hospital, and written informed consent was obtained from all subjects according to the Declaration of Helsinki.

Only untreated patients over 18 years old at diagnosis with platelet counts $<30 \times 10^{9} / \mathrm{L}$ were enrolled. ITP was diagnosed based on guidelines for ITP. ${ }^{30,31}$ Bone marrow samples were also taken from transplant donors $(n=42)$, who were considered as healthy controls. The healthy control cohort comprised 17 males and 25 females, aged 18-59 years (median, 38 years).

Patients in the group given combination therapy received 10 mg of oral ATRA twice daily and concomitant therapy with oral danazol at a relatively low dose of $200 \mathrm{mg}$ twice daily consecutively. Initial response was assessed after 4, 8 and 12 weeks of treatment. The primary endpoint of the study was overall response. Secondary endpoints were complete response, response, time to response, peak platelet count, reduction in bleeding symptoms, and safety. A complete response was defined as a platelet count of at least $100 \times 10^{9} / \mathrm{L}$. Response was defined as a platelet count between $30 \times 10^{9} / \mathrm{L}$ and $100 \times 10^{9} / \mathrm{L}$ and at least doubling of the baseline count. Overall response included complete responses and responses. No response was defined as a platelet count lower than $30 \times 10^{\%} / \mathrm{L}$ or less than doubling of the baseline count. ${ }^{31}$ Additionally, we defined time to response as the time from starting treatment to the time to achieve the response. We defined bleeding in accordance with the World Health Organization's bleeding scale $(0=$ no bleeding, $1=$ petechiae, 2 mild blood loss, $3=$ gross blood loss, and $4=$ debilitating blood loss). Lastly, we graded adverse events according to the Common Terminology Criteria for Adverse Events (version 4.0).

\section{Animal model and treatment}

All animal experiments were approved by the Ethics Committee of Peking University People's Hospital and undertaken in accordance with the Institutional Guidelines for the Care and Use of Laboratory Animals.

The inclusion and exclusion criteria for patients, the methods for MSC isolation, RNA extraction and microarrays, the immunofluorescence assays, enzyme-linked immunosorbent assays (ELISA), cell proliferation assays, apoptosis assays, western blotting, determination of CXCR4 expression on megakaryocyte surfaces, analysis of $\mathrm{CD}_{3} 4^{+}$cells, analysis of colony-forming unitmegakaryocytes, the modified monoclonal antibody-specific immobilization of platelet antigens assay, analysis of DNA methylation, real-time polymerase chain reaction analysis, gene silencing, gene overexpression, immunostaining, in situ hybridization, animal model and treatment, and statistics are explained in the Online Supplement.

\section{Results}

\section{Complement activation in the bone marrow of patients with immune thrombocytopenia}

To illuminate the role of the complement system in the bone marrow of ITP patients, we used indirect ELISA to detect the deposition of complement proteins C1q, C4d, $\mathrm{C} 3 \mathrm{~b}$ and $\mathrm{C} 5 \mathrm{~b}-9$ on the surface of MSC from ITP patients and healthy volunteers. Reference ranges of complement deposition on MSC from the healthy controls were determined: C1q ( $1.0 \pm 0.1), \mathrm{C} 4 \mathrm{~d}(0.9 \pm 0.2), \mathrm{C} 3 \mathrm{~b}(0.9 \pm 0.1)$ and C5b-9 $(1.0 \pm 0.4)$. The cutoff values defining deposition of C1q, C4d, C3b and C5b-9 were 1.2, 1.3, 1.1 and 1.8, respectively. These cutoffs represent a level of complement deposition that falls approximately two standard deviations above the reference mean for the majority of complement components. Patients were categorized as having complement activation if the level of one or more of the measured complement components was equal to or more than the cutoff value. In this study, 26 of the 58 patients were assigned to the group with complement activation (MSC-ITP-C ${ }^{+}$group), while the remaining 32 patients were 
assigned to the group without complement activation (MSC-ITP-C group). Forty-two healthy volunteers served as a control group (MSC-control group) (Online Supplementary Table S1). To further distinguish ITP patients with complement activation in bone marrow, deposition of complement proteins was also determined by indirect immunofluorescence assays (Figure 1H, I). In accordance with the results of the indirect ELISA, MSC from ITP patients in the MSC-ITP-C ${ }^{+}$group were evidently stained with the complement components, especially C4d and C5b-9 (Online Supplementary Figure S1).

Intrinsic mRNA alterations in complement-activated mesenchymal stem cells from patients with immune thrombocytopenia

To investigate whether the MSC from patients in the
MSC-ITP-C ${ }^{+}$group show transcriptome abnormalities and to better understand molecular pathways that may regulate MSC-ITP-C ${ }^{+}$biology, we performed an mRNA expression analysis by microarray.

Unsupervised clustering analysis comparing MSC-ITP$\mathrm{C}^{+}$to MSC-ITP-C and MSC-control showed distinctive gene expression signatures and we observed differential expression (fold change $>2, P<0.05$ ) of 2,978 probes between the groups, with 1,926 upregulated and 1,052 downregulated (Figure 1A). Bioinformatics analysis was used to identify complement, IL-1 family, IL-1 receptor family, CXC chemokine family, CXC chemokine receptor family, tumor necrosis factor (TNF) family and TNF receptor family-related genes that were differentially expressed in the MSC-ITP-C ${ }^{+}$group (fold change $>2.0, P<0.05$ ) (Online Supplementary Figure S2A-D).
A

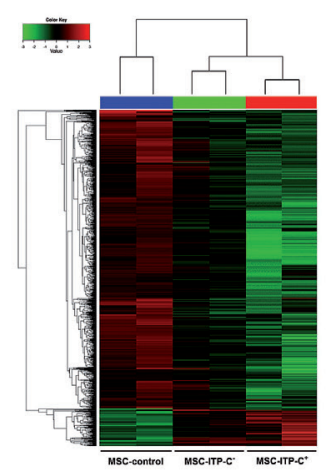

$E$
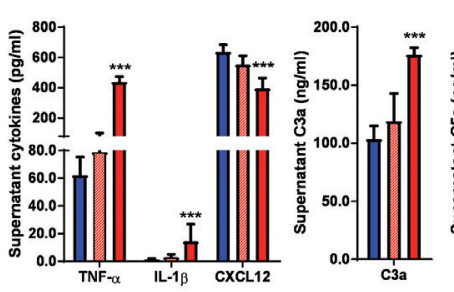

H
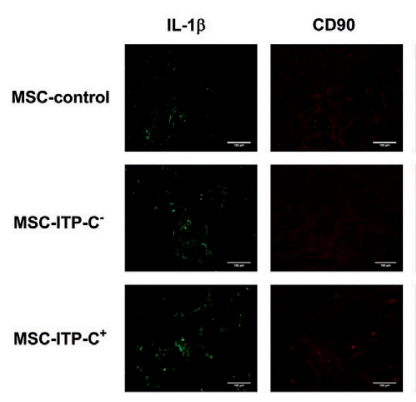

B

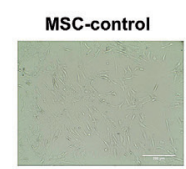

D

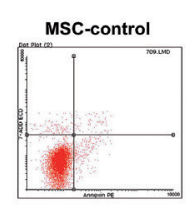

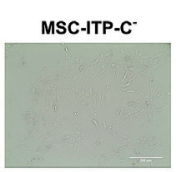

MSC-ITP-C

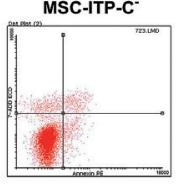

C
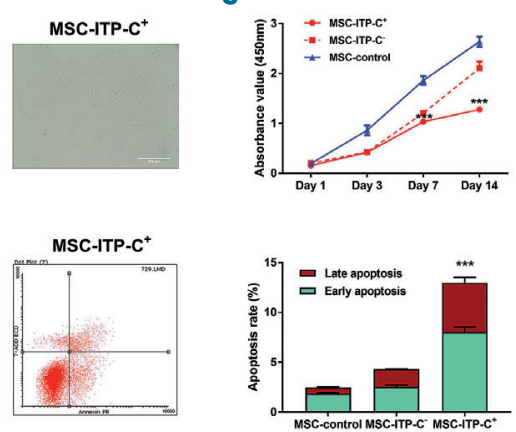

$\mathrm{F}$

G
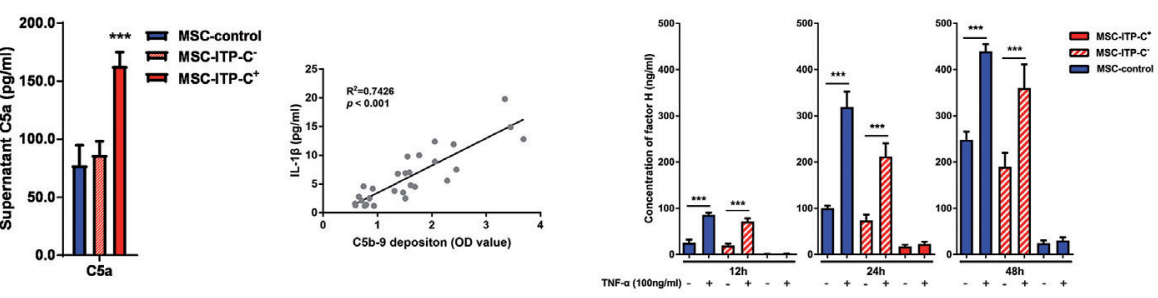

I
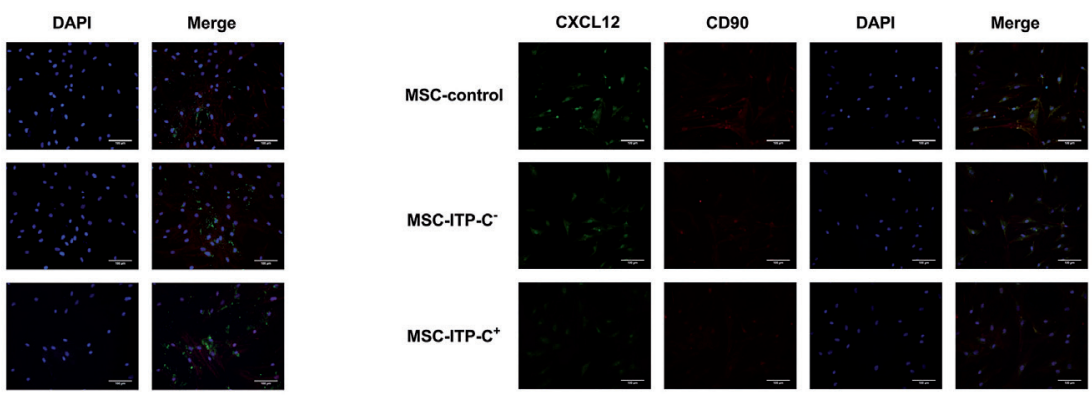

Figure 1. Mesenchymal stem cells with complement deposition from patients with immune thrombocytopenia are genetically and functionally abnormal. (A) Unsupervised clustering analysis of differentially selected probes. (B) Morphology of mesenchymal stem cells (MSC) from the three groups (MSC-ITP-C+: MSC from patients with immune thrombocytopenia with complement deposition on MSC; MSC-ITP-C MSC from patients with immune thrombocytopenia without complement deposition on MSC; MSC-control: MSC from healthy subjects) under a light microscope (original magnification $\times 3200$; scale bar: $200 \mu \mathrm{m})$. (C) The growth curves of the MSC-ITP-C ${ }^{+}(n=26)$, MSC-ITP-C $(n=32)$ and MSC-control $(n=42)$ groups at passage 3 from four independent experiments. One-way analysis of variance (ANOVA). (D) Cell apoptosis of MSC determined by annexin V assays (MSC-ITP-C $\mathrm{C}^{+} \mathrm{n}=12$; MSC-ITP-C, $n=21$; MSC-control, $n=12$; one-way ANOVA). (E) The levels of tumor necrosis factor- $\alpha$ (TNF- $\alpha$ ), interleukin-1 $\beta$ (IL-1 $\beta$ ), CXCL12, and the complement factors C3a and C5a in bone marrow supernatants from the MSC-control ( $n=42$ ), MSC-ITP-C $(n=32)$ and MSC-ITP- $C^{+}(n=26)$ groups; one-way ANOVA). $(F)$ The correlation between the level of IL-1 $\beta$ in culture supernatants and C5b-9 deposition on MSC $\left(R^{2}=\right.$ $0.7426, P<0.001$, Spearman rank correlation rho). (G) Levels of factor $\mathrm{H}$ in MSC culture supernatants 12,24 , and $48 \mathrm{~h}$ after co-culture with TNF- $\alpha$, determined by enzyme-linked immunosorbent assays (paired t-tests). ( $\mathrm{H}, \mathrm{I})$ Intracellular expression of IL-1 $\beta$ and CXCL12 in MSC-ITP-C $\mathrm{C}^{+}$, MSC-ITP-C and MSC-control, determined by immunofluorescence assays. Scale bar: $100 \mu \mathrm{m}$. DAPI: 4',6-diamidino-2-phenylindole. 
A

\section{MSC-control}

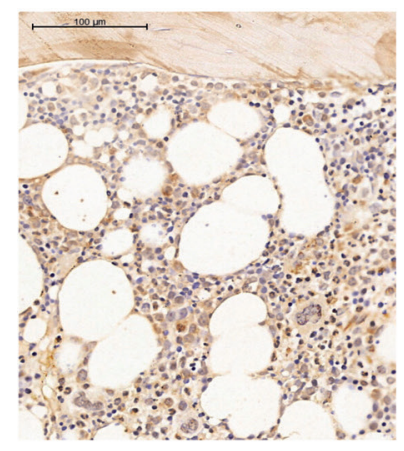

B

MSC-ITP-C-

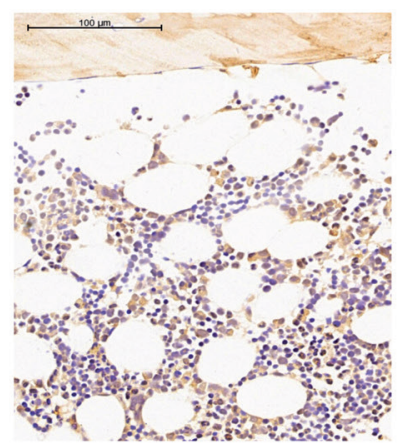

C

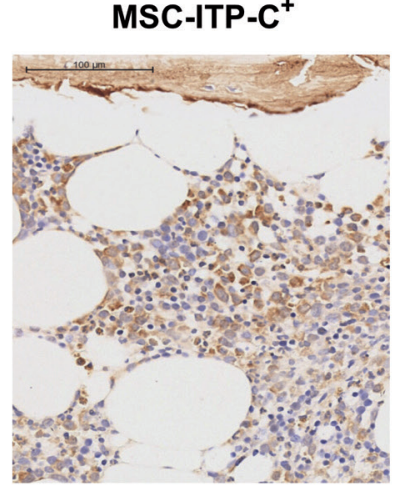

D

Bone-associated marrow
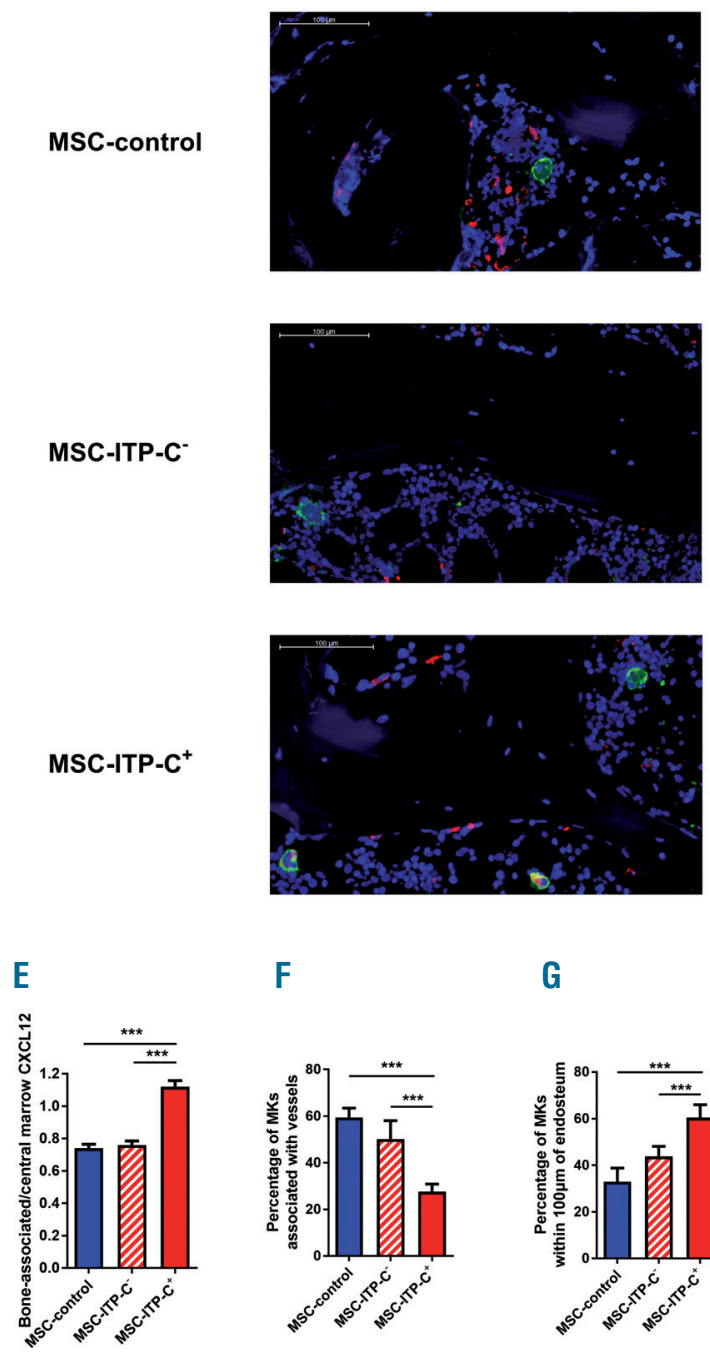

$\mathbf{F}$

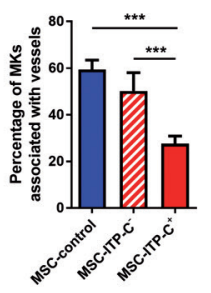

G

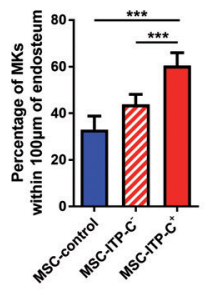

Central marrow
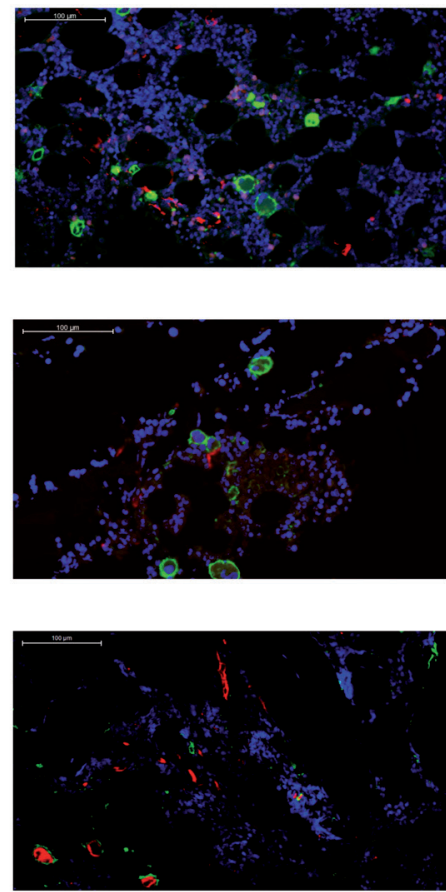

H

I
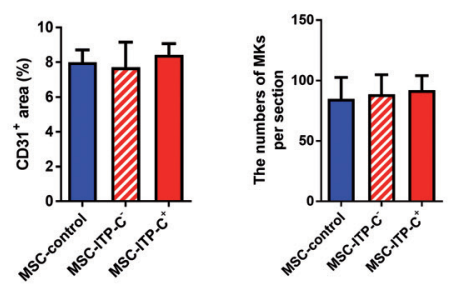

Figure 2. Altered CXCL12 gradients and megakaryocyte marrow niche occupancy in immune thrombocytopenia patients with complement deposition on their mesenchymal stem cells. (A-C) Representative images of radioactive in situ hybridization with CXCL12 antisense probe [CXCL12 transcripts: brown pseudocolor; 4',6diamidino-2-phenylindole (DAPI): blue] on bone marrow sections from the three groups [MSC-control: mesenchymal stem cells (MSC) from healthy subjects; MSCITP-C: MSC from patients with immune thrombocytopenia (ITP) without complement deposition on MSC; MSC-ITP-C+: MSC from patients with ITP with complement deposition on MSC]. Scale bar: 100 um. (D) Representative images of marrow immunohistochemistry for CD41 (megakaryocytes, green) and CD31 (vascular endothelium, red) from the MSC-control, MSC-ITP-C and MSC-ITP-C ${ }^{+}$groups (left: bone-associated marrow; right: central marrow). Scale bar: 100 um. (E) Ratio of CXCL12 transcript area in the bone-associated region (between 0-100 $\mu \mathrm{m}$ from the endosteal surface within the diaphysis) compared to an immediately adjacent region of the same size (between 100-200 $\mu \mathrm{m}$ from the endosteal surface) for ITP patients (MSC-ITP-C and MSC-ITP-C+) and healthy volunteers (MSC-control). (F) Quantification of CD41 $1^{+}$megakaryocytes (MKs) physically associated with $\mathrm{CD} 31^{+}$vessels (between 0-10 $\mu \mathrm{m}$ from the sinusoidal endothelium within the diaphysis) by immunohistochemistry. (G) Quantification of $\mathrm{CD} 41^{+}$megakaryocytes in the bone-associated region (within $100 \mu \mathrm{m}$ of the endosteal surface within the diaphysis) by immunohistochemistry. (H) Quantification of vessel densities by immunofluorescence staining for CD31. (I) Quantification of CD41 ${ }^{+}$megakaryocytes per section. (EI) MSC-control, $n=36$; MSC-ITP-C, $n=36$; MSC-ITP-C $C^{+}, n=36$; one-way analysis of variance). 
To evaluate the regulation of MSC-ITP-C ${ }^{+}$on cell signaling, we performed a canonical pathway analysis. Canonical pathway annotation enabled us to assign differentially expressed genes to 28 pathways. The first nine enriched pathways were the unfolded protein response, protein ubiquitination pathway, cell cycle, IL-1 signaling, p53 signaling, endoplasmic reticulum stress pathway, ERK5 signaling, NRF2-mediated oxidative stress response, and p38 MAPK signaling, which might convey the differences between MSC-ITP-C+, MSC-ITP-C; and MSC-control. The first 12 enriched pathways are shown in Online Supplementary Figure S2E. Disease and functional heatmaps revealed the activated or inhibitory relationship between differentially expressed genes and diseases and functions (Online Supplementary Figure S2F). "Organismal death" (Z-score $=16.497)$ and "Morbidity or mortality" (Z-score $=16.484)$ were significantly activated in MSC-ITP-C+.

\section{Complement-activated mesenchymal stem cells from immune thrombocytopenia patients showed increased apoptosis and functional impairment}

Bone marrow MSC were successfully isolated. Flow cytometry analysis demonstrated that MSC from both healthy donors and ITP patients expressed CD105, CD73, and CD90 and lacked expression of CD14, CD19, CD34, CD45, and HLA-DR (Online Supplementary Figure S3). MSC-control expanded and acquired a spindle shape morphology during culture. In contrast, MSC-ITP-C ${ }^{+}$ expanded more slowly and appeared larger and flattened (Figure 1B). CCK8 proliferative assays were conducted on MSC at days 1, 3, 7 and 14 after the third passage. The growth curves showed a lower proliferative capacity of MSC-ITP-C + (Figure 1C). We further assessed the apoptotic cell rate using annexin $\mathrm{V}$. As shown in Figure 1D, the rates of both early apoptosis and late apoptosis were higher in the MSC-ITP-C $\mathrm{C}^{+}$group.

Since the complement system has been shown to be associated with the inflammatory response, we determined cytokine levels in bone marrow supernatants from ITP patients and healthy controls using ELISA. The levels of the inflammatory factors IL-1 $\beta$ and TNF- $\alpha$ were both significantly higher $(P<0.001)$ in the MSC-ITP- $C^{+}$group compared with the MSC-ITP-C and MSC-control groups (Figure 1E), while the levels of CXCL12 were significantly lower $(P<0.001)$ (Figure 1E). Intracellular expression of IL$1 \beta$ and CXCL12 was further confirmed by immunofluorescence assays (Figure 1H, I). Moreover, the levels of expression of complement activation fragments $\mathrm{C} 3 \mathrm{a}$ and C5a were also significantly higher $(P<0.001$ for both) in MSC-ITP-C ${ }^{+}$(Figure 1E). There was a positive correlation between the level of IL-1 $\beta$ in culture supernatant and C5b-9 deposition on MSC $\left(\mathrm{R}^{2}=0.7426, P<0.001\right.$, Spearman rank correlation rho) (Figure 1F).

In view of the diminished expression of complement factor $\mathrm{H}$ found by microarray analysis in the MSC-ITP-C ${ }^{+}$ group, we performed a TNF- $\alpha$ stimulation test. Factor $\mathrm{H}$ concentrations in MSC culture supernatants 12,24 , and $48 \mathrm{~h}$ after co-culture with TNF- $\alpha$ were measured by ELISA. Upon exposure to TNF- $\alpha$, no significant effect on the secretion of factor $\mathrm{H}$ was seen in the MSC-ITP-C $\mathrm{C}^{+}$ group, which was not consistent with the significantly increased factor $\mathrm{H}$ secretion observed in the MSC-ITP-Cand MSC-control groups (Figure 1G).
Altered CXCL12 gradients and megakaryocyte marrow niche occupancy in immune thrombocytopenia patients with mesenchymal stem cell complement deposition

As the MSC-ITP-C ${ }^{+}$group showed attenuated expression of both CXCL12 protein and its encoding gene, we investigated whether there are changes in bone marrow CXCL12 and their consequences for megakaryocytes. Given the importance of the location of CXCL12 production for this chemokine's chemotactic function ${ }^{32}$ and the varied injury and recovery kinetics of different bone marrow cell populations in MSC-ITP-C ${ }^{+}$, we determined the location of CXCL12 transcripts in bone marrow sections of the MSC-ITP-C+, MSC-ITP-C and MSC-control groups by radioactive in situ hybridization (Figure 2A-C). As expected, the MSC-ITP-C ${ }^{+}$group showed an overall increase in the CXCL12 message in the bone-associated marrow and a decrease in the central marrow (Figure 2C). Differences in the distribution of CXCL12 in the bone marrow were quantified by comparing CXCL12 transcript levels within the bone-associated region to the levels in an adjacent region within the central marrow (Figure 2E). Interestingly, we detected the development of a CXCL12 gradient toward the endosteum in the MSCITP-C ${ }^{+}$group (Figure $2 \mathrm{E}$ ). Since megakaryocytes interact with sinusoidal endothelium, we examined the effects of observed shifts of CXCL12 expression on marrow vasculature by immunohistochemistry (Figure 2D). A noticeable, marked decrease in megakaryocytes associated with sinusoids was observed in the MSC-ITP- $\mathrm{C}^{+}$group (Figure 2D, F). Given the development of a CXCL12 gradient toward the endosteum in the MSC-ITP-C ${ }^{+}$group, we also quantified megakaryocyte distribution in the bone-associated region, defined as the region within $100 \mu \mathrm{m}$ of the diaphyseal endosteum. ${ }^{33}$ Strikingly, there was an increase in megakaryocytes in the bone-associated marrow, coincident with the increased CXCL12 gradient (Figure 2D, G). Altogether, these results implicated spatial and temporal alterations in marrow CXCL12 of the MSC-ITP-C group in the observed changes in megakaryocyte niche occupancy. The overall numbers of megakaryocytes and the density of vessels were not significantly different between the MSC-control, MSC-ITP-C and MSC-ITP-C+ groups (Figure $2 \mathrm{H}, \mathrm{I}$ ).

\section{The C5b-9/interleukin-1 $\beta$ loop regulates mesenchymal} stem cells from patients with immune thrombocytopenia

On the basis of the finding of enhanced expression of IL-1 $\beta$ in the MSC-ITP-C ${ }^{+}$group, we hypothesized that IL$1 \beta$ induced by complement activation plays an important role in the dysfunction of MSC. The production of proIL-1 $\beta$ and caspase- 1 was significantly higher in MSC-ITP$\mathrm{C}^{+}$than in MSC-ITP-C and MSC-control (Figure 3A), indicating that complement induced IL-1 $\beta$ maturation and caspase-1 processing.

To obtain further insight into the mechanisms underlying the increased expression of IL-1 $\beta$, we then analyzed the role of signaling pathways in MSC from ITP patients and healthy volunteers. Expression of the interleukin-1 receptor (IL-1R) was upregulated on MSC-ITP-C+ (Figure 3B) and increased activation of MyD88 and NF-KB (p65) was detected in MSC-ITP-C+ (Figure 3C). Simultaneously, the expression levels of $\mathrm{p}$-ERK1/2 and p-p38 MAPK were significantly higher in MSC-ITP-C ${ }^{+}$than in the other two 
groups (Figure 3D). Collectively, these data indicate the involvement of IL-1R/MyD88/NF-kB, ERK1/2 and p38 MAPK signaling pathways in MSC-ITP-C ${ }^{+}$.

To determine whether the autocrine IL- $1 \beta$ protein is crucial for the dysfunction of MSC, MSC-control were transfected with IL-1 $\beta$-cDNA and signal sequences of IL$1 \mathrm{R}$ antagonist. The secretion of IL-1 $\beta$ was markedly improved in MSC cell lysates on day 7, while CXCL12 expression was inhibited (Figure 3E, F). Moreover, phosphorylation of MyD88, ERK1/2, p38 MAPK and NF-кB was enhanced (Figure 3I). Lentiviruses carrying IL-1 $\beta$ -
siRNA were transfected into MSC-ITP-C C $^{+}$The expression of IL- $1 \beta$ was markedly reduced in the cell lysates on day 7 (Figure 3G). Conversely, CXCL12 expression was upregulated (Figure $3 \mathrm{H}$ ). IL-1 $\beta$ knockdown decreased the phosphorylation of MyD88, ERK1/2, p38 MAPK and NF$\mathrm{\kappa B}$ (Figure 3I). As expected, CCK8 proliferative assays at days 1,3 and 7 revealed altered proliferative capacity (Figure 3J, K). The apoptosis rate was higher in MSC-control transfected with IL-1 $\beta$ cDNA and lower in MSC-ITP$\mathrm{C}^{+}$with IL-1 $\beta$-siRNA (Figure $3 \mathrm{~L}, \mathrm{M}$ ). Furthermore, bone marrow sections from patients in the MSC-ITP- $\mathrm{C}^{+}$group
A
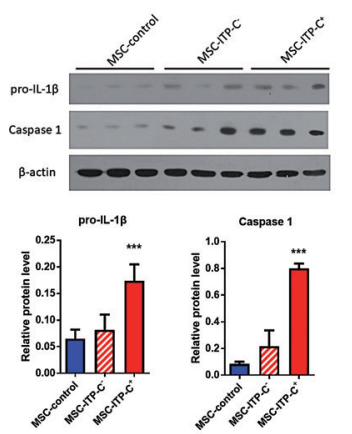

$E$

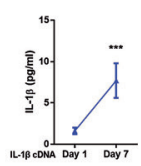

$\mathrm{F}$

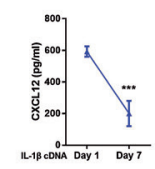

G

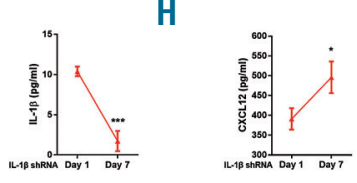

J

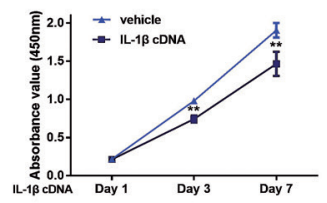

K

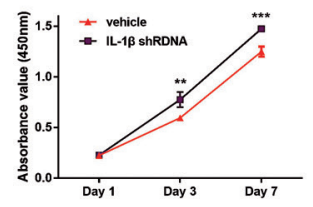

B
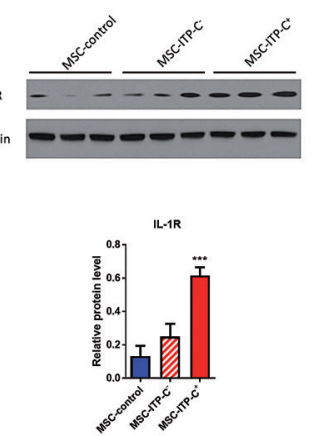

C
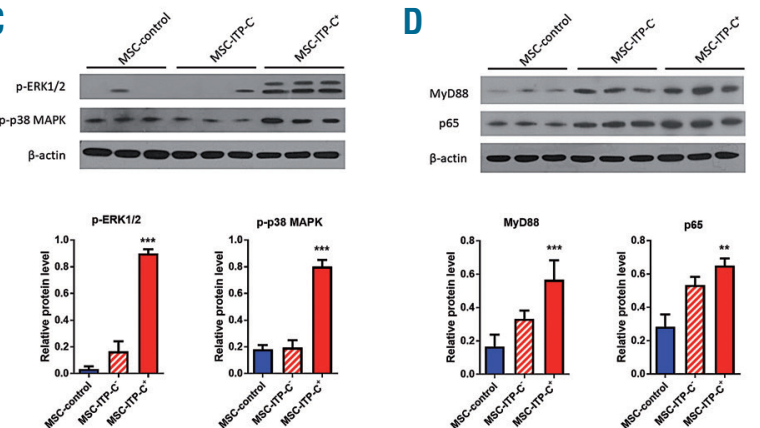

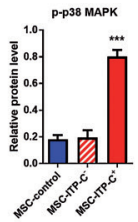

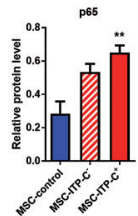

I

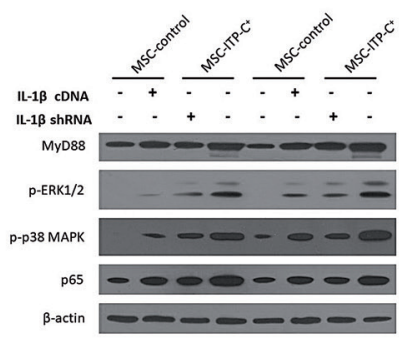

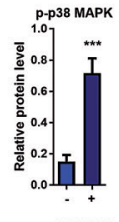

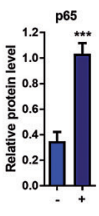

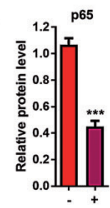

$\mathbf{L}$
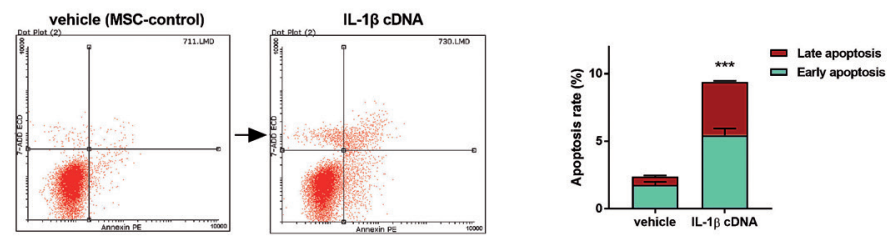

M
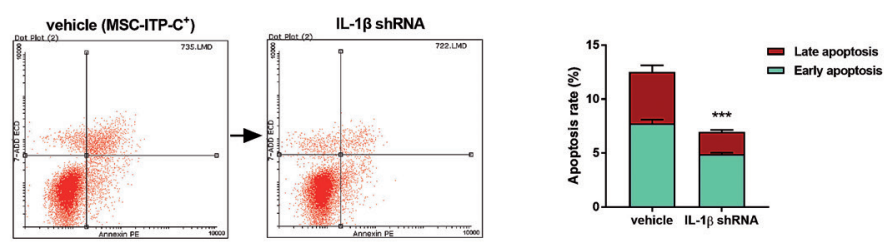

Figure 3. The C5b-9/interleukin-1 $\beta$ loop regulates mesenchymal stem cells from patients with immune thrombocytopenia. (A, B) Levels of pro-interleukin-1 $\beta$ (IL$1 \beta$ ) and caspase 1 (A) and interleukin-1 receptor (IL-1R) (B) in the three groups [MSC-control: mesenchymal stem cells (MSC) from healthy subjects; MSC-ITP-C MSC from patients with immune thrombocytopenia (ITP) without complement deposition on MSC; MSC-ITP-C : MSC from patients with ITP with complement deposition on MSC] were detected by western blotting in MSC cell lysates. $\beta$-actin was used as the loading control (MSC-control, $n=12 ;$ MSC-ITP-C, $n=12$; MSC-ITP-C ${ }^{+}, n=12$; One-way analysis of variance (ANOVA). (C, D) Phosphorylation of MyD88, NF-KB, ERK1/2 and p38 MAPK signaling pathway proteins in MSC from ITP patients and healthy volunteers. $\beta$-actin was used as the loading control (MSC-control, $n=12$; MSC-ITP-C, $n=12$; MSC-ITP-C ${ }^{+}, n=12$; one-way ANOVA). (E, F) Expression of IL-1 $\beta$ and CXCL12 in MSC-control cell lysates at day 1 and day 7 with transfection with lentivirus carrying the IL-1 $\beta$-cDNA from two independent experiments ( $\mathrm{n}=12$; Student $t$-tests). (G, H) Expression of IL-1 $\beta$ and CXCL12 in MSC-ITP-C ${ }^{+}$cell lysates 7 days after transfection with lentivirus carrying the IL-1 $\beta$-siRNA from two independent experiments ( $n=12$; Student $t$-tests). (I) Phosphorylation of MyD88, ERK1/2, p38 MAPK and NF-KB in transfected MSC by Western blotting ( $n=8$; Student $t$-tests). (J, K) CCK8 proliferative assays of transfected MSC from three independent experiments (MSC-control, $n=12$; MSC-ITP-C ${ }^{+}, \mathrm{n}=12$; Student $t$-tests). (L, M) Apoptosis rate of transfected MSC (MSC-control, $n=12 ; M^{2}$-ITP-C ${ }^{+}, n=12 ; \chi^{2}$ tests). 
revealed increased intensity of IL- $1 \beta$ fluorescence and number of TUNEL ${ }^{+} \mathrm{CD} 90^{+} \mathrm{MSC}$ in the central marrow (Online Supplementary Figures $S 4 A$ and S5A). These results imply that the C5b-9/IL-1 $\beta$ loop contributes to the dysfunction of MSC-ITP-C ${ }^{+}$.
All-trans retinoic acid directed in vitro functional recovery of complement-activated mesenchymal stem cells from patients with immune thrombocytopenia

We previously reported the therapeutic efficacy of ATRA in ITP patients. ${ }^{27}$ In order to ascertain the mecha-
A

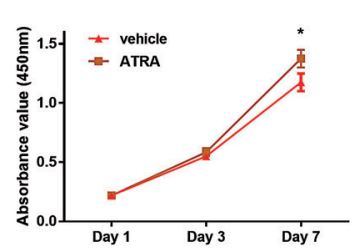

C

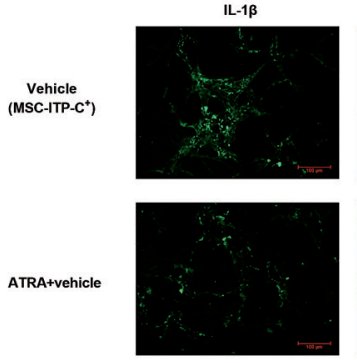

B

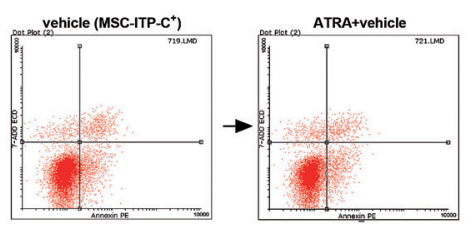

D

DAPI
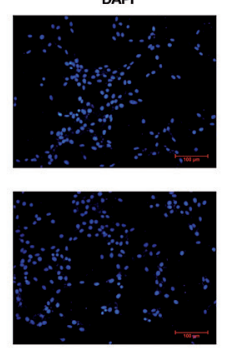

Merge
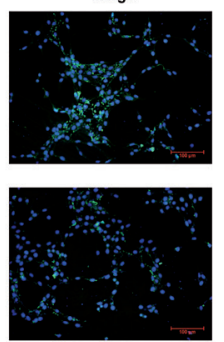
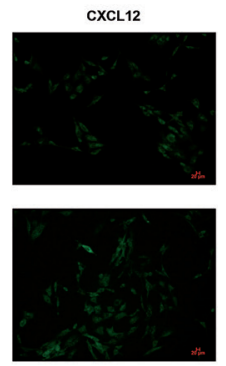
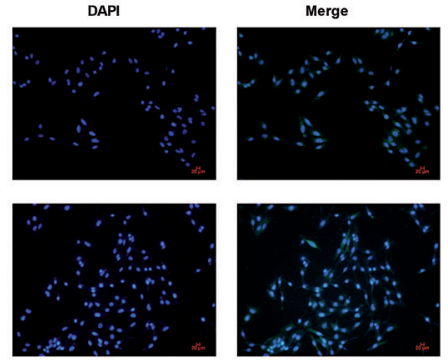

E

$\mathbf{F}$
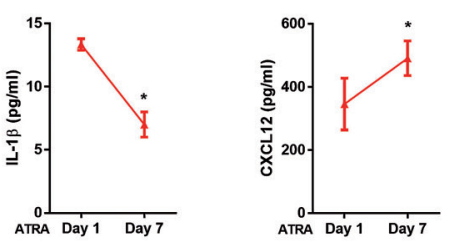

G
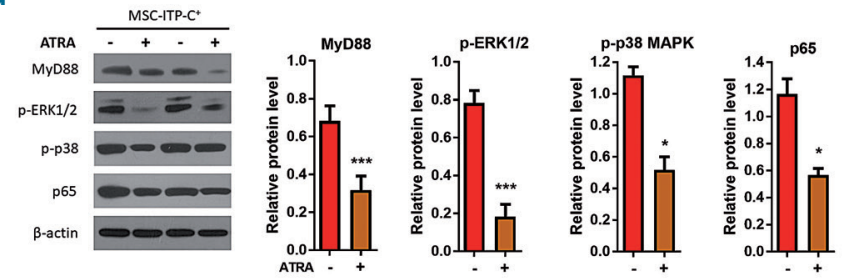

$\mathrm{H}$

I
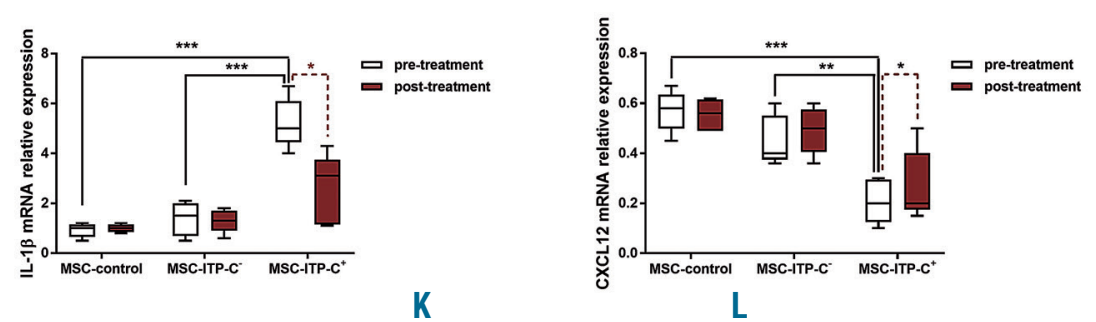

J

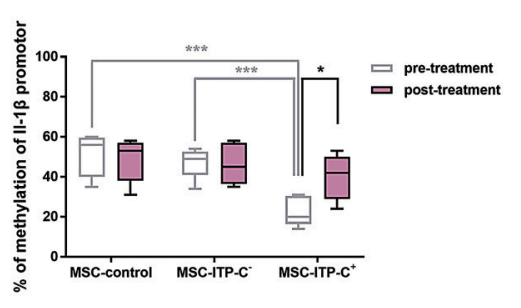

K
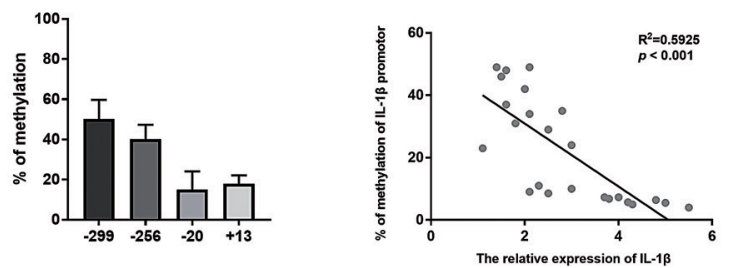

Figure 4. All-trans retinoic acid directed in vitro functional recovery of mesenchymal stem cells from immune thrombocytopenia patients with complement deposition on their mesenchymal stem cells. (A) CCK8 proliferative assays of all-trans retinoic acid (ATRA) treated mesenchymal stem cells (MSC) from patients with immune thrombocytopenia (ITP) with complement deposition on their MSC (MSC-ITP-C ${ }^{+}$) from three independent experiments ( $\mathrm{n}=12$; Student $t$-tests). (B) Apoptosis rate of ATRA-treated MSC-ITP-C ${ }^{+}\left(n=12\right.$; Student $t$-tests). (C, D) Intracellular expression of interleukin-1 $\left(\right.$ IL-1 $\beta$ ) and CXCL12 in untreated and treated MSC-ITP-C ${ }^{+}$by immunofluorescence assays. (E, F) The levels of IL-1 $\beta$ and CXCL12 in MSC-ITP-C ${ }^{+}$cell lysates at day 1 and day 7 with the administration of ATRA from two independent experiments ( $n=12$; Student $t$-tests). (G) Phosphorylation of MyD88, ERK1/2, p38 MAPK and NF-KB in MSC-ITP- $C^{+}$before and after treatment with ATRA by western blotting ( $n=8$; Student $t$-tests). (H, I) mRNA levels of IL-1 $\beta$ and CXCL12 in MSC from healthy controls (MSC-control), MSC from ITP without complement deposition on their MSC (MSC-ITP-C) and MSC-ITP-C ${ }^{+}$before and after treatment with ATRA. mRNA levels of IL-1 $\beta$ and CXCL12 were normalized to the mRNA levels of 18srRNA $(n=12$ independent experiments). Tukey tests for multiple comparisons among pre-treatment groups. Student $t$-tests for the comparisons between pre-treatment and post-treatment groups from independent experiments. (J) Promoter DNA methylation of IL-1 $\beta$ in MSC-control, MSC-ITP-C and MSC-ITP-C $\mathrm{C}^{+}$before and after treatment with ATRA. Percentages of DNA methylation were obtained by analyzing the mean methylation of all the CpG sites present in the promoter ( $\mathrm{n}=12$ independent experiments). Tukey tests for multiple comparisons among pre-treatment groups. $\chi^{2}$ tests for the comparisons between pre-treatment and post-treatment groups from independent experiments. (K) The methylation status of $-299,-256,-20$ and $+13 \mathrm{CpG}$ sites in the IL-1 $\beta$ proximal promoter ( $\mathrm{n}=12$ independent experiments). (L) There was a linear relationship between DNA methylation of the IL-1 $\beta$ promoter and mRNA levels $\left(R^{2}=0.5925, P<0.001, S p e a r m a n\right.$ rank correlation rho). 
nisms underlying the response to ATRA, MSC from ITP patients and healthy volunteers were treated with ATRA. As was foreseeable, exposure of MSC-ITP-C ${ }^{+}$to ATRA led to marked proliferation and a reduced rate of apoptosis (Figure 4A, B). Downregulated secretion of IL-1 $\beta$ and upregulated levels of CXCL12 were found in the treated MSC-ITP-C ${ }^{+}$group (Figure 4C, D). Levels of IL-1 $\beta$ and CXCL12 in MSC cell lysates were regulated accordingly (Figure 4E, F). Distinctly, the treated group showed inhibited phosphorylation of MyD88, ERK1/2, p38 MAPK and NF-кB (Figure 4G). No significant differences in proliferative capacity, apoptosis rate, cytokine secretion and signaling pathways were observed in either the MSC-ITP-Cgroup (Online Supplementary Figure S6) or the MSC-control group (Online Supplementary Figure S7) before or after the administration of ATRA.

We further examined the potential genetic mechanism of the suppressive effect of ATRA on IL-1 $\beta$ in MSC-ITP$\mathrm{C}^{+}$. Analysis of the expression of the gene encoding IL-1 $\beta$ showed a dramatic, significant expression of IL-1 $\beta$ mRNA levels by MSC-ITP-C ${ }^{+}$(Figure $4 \mathrm{H}$ ). ATRA produced a modest decrease in IL-1 $\beta$ mRNA expression of MSC-ITP$\mathrm{C}^{+}$, while it had no effect on IL-1 $\beta$ mRNA levels of MSCITP-C and MSC-control (Figure 4H). CXCL12 mRNA levels were downregulated in MSC-ITP-C ${ }^{+}$and could be increased by the administration of ATRA (Figure 4I). The frequency of DNA hypermethylation of the IL-1 $\beta$ promoter in MSC-ITP-C ${ }^{+}$was significantly lower than that in MSC-ITP-C and MSC-control (Figure 4J). ATRA-treated MSC-ITP-C ${ }^{+}$had higher DNA methylation of the IL-1 $\beta$ promoter (Figure 4J). We further analyzed the percentage methylation at the $-299,-256,-20$ and +13 CpG sites in the IL-1 $\beta$ promoter (Figure 4K). The percentage methylation was $50 \%, 40 \%, 15 \%$, and $18 \%$ at the $-299,-256,-20$,
A

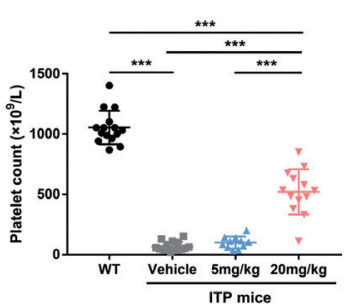

D

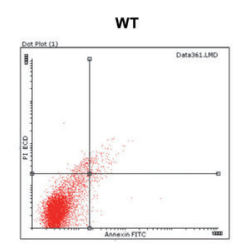

E

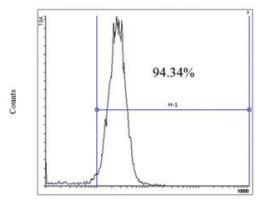

CD29
B

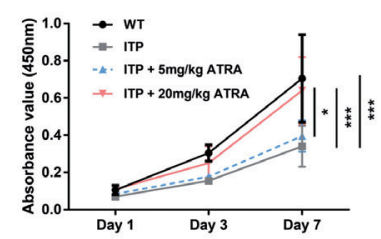

C

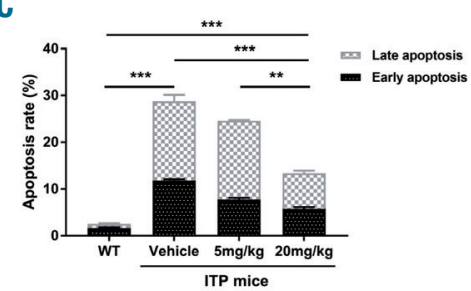

ITP mice
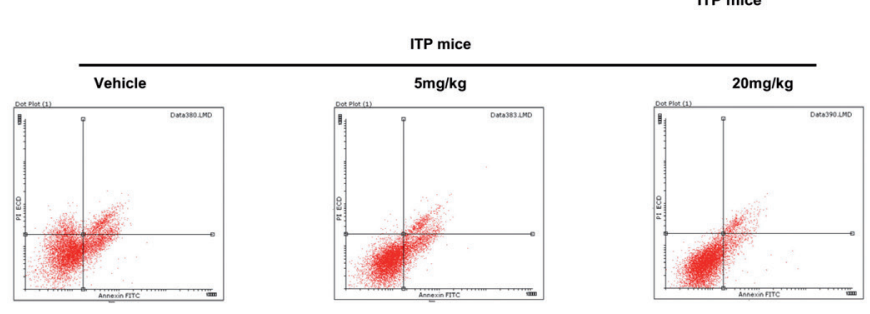

E

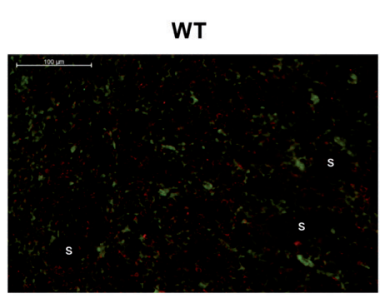

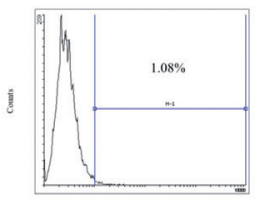

CD45

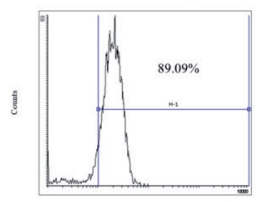

CD105

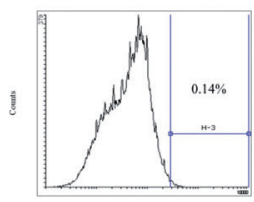

CD34

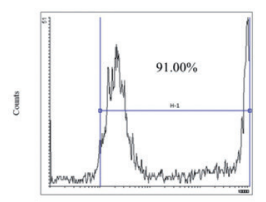

$\operatorname{cD} 90$
G

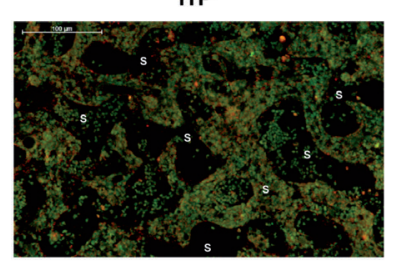

Figure 5. All-trans retinoic acid directed functional recovery of bone marrow mesenchymal stem cells from mice with immune thrombocytopenia. (A) Peripheral platelet counts of wildtype (WT) mice $(n=15)$, immune thrombocytopenia (ITP) mice $(n=15)$, ITP mice treated with a 5 mg/kg intraperitoneal injection of all-trans retinoic acid (ATRA) $(n=11)$ and ITP mice treated with a $20 \mathrm{mg} / \mathrm{kg}$ intraperitoneal injection of ATRA $(n=13)$. Tukey tests. (B) The growth curves of bone marrow mesenchymal stem cells (MSC) from WT mice $(n=6)$, ITP mice $(n=6)$, ITP mice treated with ATRA $5 \mathrm{mg} / \mathrm{kg}$ intraperitoneal injection ( $\mathrm{n}=6$ ) and ITP mice treated with ATRA $20 \mathrm{mg} / \mathrm{kg}$ intraperitoneal injection $(n=6)$ at passages 7-12. Covariance analyses. (C, D) Cell apoptosis of bone marrow MSC determined by annexin $V$ assays (WT mice, $n=6$; ITP mice, $n=6$; ITP mice treated with ATRA $5 \mathrm{mg} / \mathrm{kg}$ intraperitoneal injection, $\mathrm{n}=6$; ITP mice treated with ATRA $20 \mathrm{mg} / \mathrm{kg}$ intraperitoneal injection, $\mathrm{n}=6$; Tukey tests). (E) The phenotypes of mice bone marrow MSC identified by flow cytometer. (F) C5b-9 deposition (green) assayed by marrow immunohistochemistry of WT mice and ITP mice (CD31, vascular endothelium, red). S, sinusoid. Scale bar: $0.1 \mathrm{~mm}$. (G) The levels of interleukin-1 $\beta$ (IL-1 $\beta$ ) and CXCL12 in bone marrow supernatants (WT mice, $n=6$; ITP mice, $n=6$; ITP mice treated with ATRA $5 \mathrm{mg} / \mathrm{kg}$ intraperitoneal injection, $\mathrm{n}=6$; ITP mice treated with ATRA $20 \mathrm{mg} / \mathrm{kg}$ intraperitoneal injection, $\mathrm{n}=6$; Tukey tests). (Continued on next page) 
and +13 CpG sites, respectively. DNA methylation of the IL-1 $\beta$ promoter and mRNA levels were inversely correlated $\left(R^{2}=0.5925, P<0.001\right.$, Spearman rank correlation rho) (Figure $4 \mathrm{~L}$ ). Above all, in the presence of ATRA, IL-1 $\beta$ expression in MSC-ITP-C ${ }^{+}$was alleviated and this modification was associated with hypermethylation.

\section{All-trans retinoic acid directed the functional recovery} of bone marrow mesenchymal stem cells of mice with immune thrombocytopenia

$\mathrm{CD} 1^{+} \mathrm{SCID}$ mice engrafted with $5 \times 10^{4}$ splenocytes from CD61 knockout (KO) mice immunized against $\mathrm{CD} 1^{+}$-platelets exhibited profound thrombocytopenia ${ }^{34}$ (Online Supplementary Figure S8) and some animals died of bleeding within 2-3 weeks after transfer. Platelet counts were significantly lower in ITP mice than in wild-type (WT) mice $\left(68.73 \pm 38.76 \times 10^{9} / \mathrm{L}\right.$ vs. $1054.07 \pm 138.93 \times 10^{\circ} / \mathrm{L}$, respectively; $P=0.000$ ) (Figure $5 \mathrm{~A}$ ). The phenotypes of mice bone marrow MSC were determined by flow cytometry. The results showed that these cells were homogenously positive for the mesenchymal markers CD29, CD90 and CD105, but negative for the hematopoi-
Bone-associated marrow
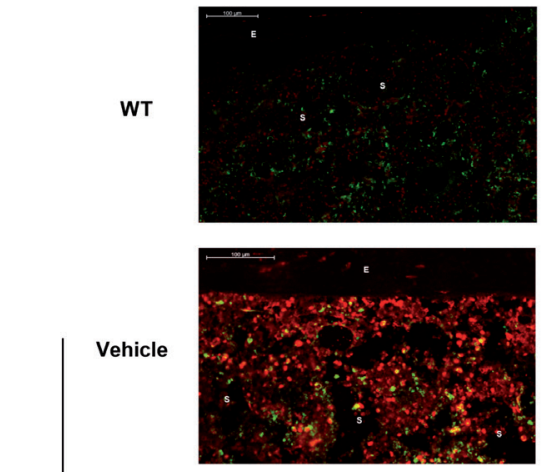

Vehicle
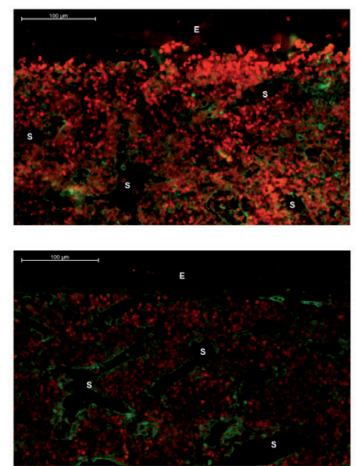

Central marrow
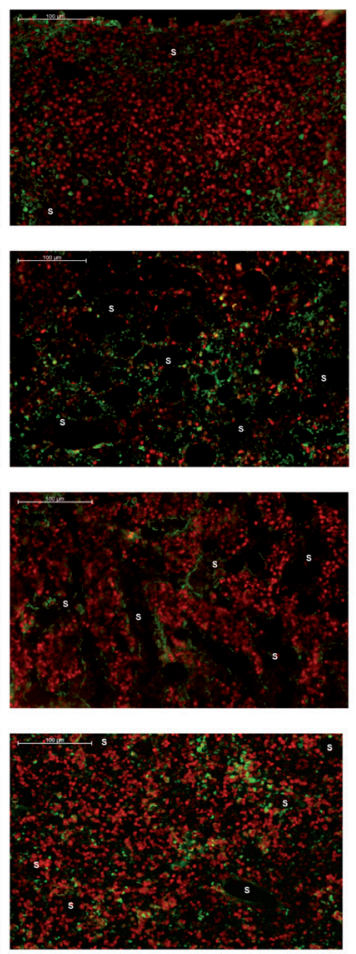

Bone-associated marrow
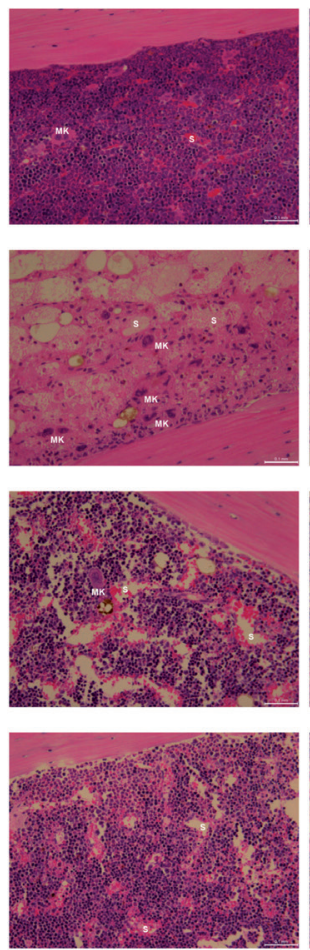

Central marrow
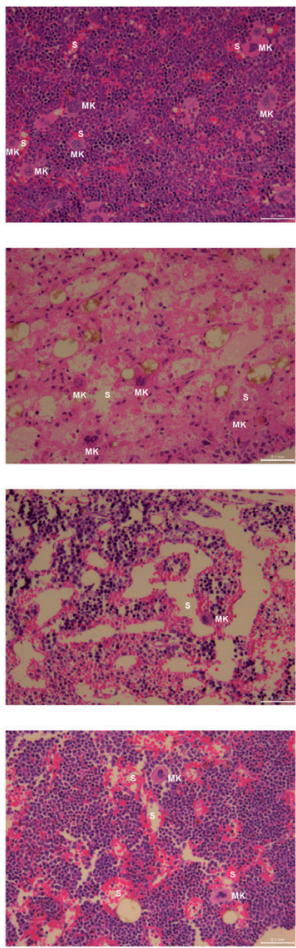

I

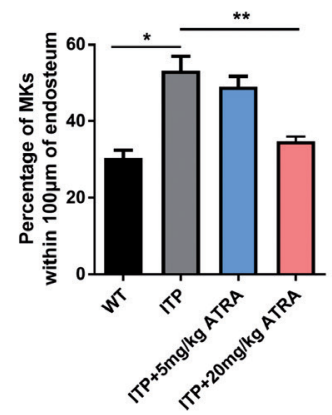

J

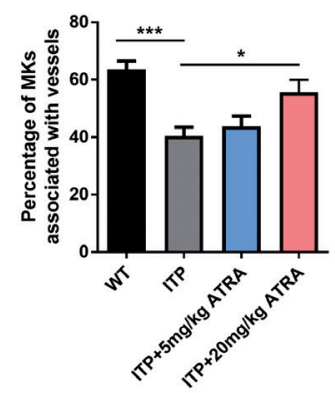

K

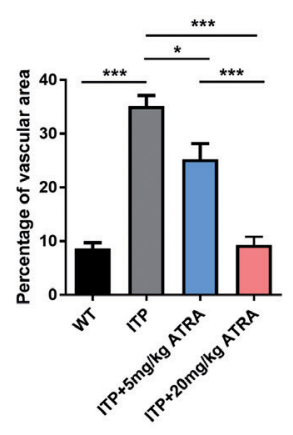

Figure 5. (H) Left: expression of CXCL12 (red) and CD31 (vascular endothelium, green) in the bone marrow niche assayed by marrow immunohistochemistry of WT mice, ITP mice, ITP mice treated with ATRA $5 \mathrm{mg} / \mathrm{kg}$ intraperitoneal injection and ITP mice treated with ATRA $20 \mathrm{mg} / \mathrm{kg}$ intraperitoneal injection. Right: Hematoxylin \& eosin staining of marrow sections from WT mice, ITP mice, ITP mice treated with $5 \mathrm{mg} / \mathrm{kg}$ intraperitoneal injection and ITP mice treated with 20 mg/kg intraperitoneal injection. S, sinusoid; E, endosteum; MK, megakaryocyte. Scale bar: $0.1 \mathrm{~mm}$. (I) Quantification of megakaryocytes in the bone-associated region (within 100 $\mu \mathrm{m}$ of the endosteal surface within the diaphysis) by immunohistochemistry (WT mice, $\mathrm{n}=27$; ITP mice, $\mathrm{n}=27$; ITP mice treated with ATRA 5 mg/kg intraperitoneal injection, $n=15$; ITP mice treated with ATRA $20 \mathrm{mg} / \mathrm{kg}$ intraperitoneal injection, $\mathrm{n}=21$ ). (Continued from previous page). (J) Quantification of megakaryocytes physically associated with sinusoids (between 0-10 $\mu \mathrm{m}$ from the sinusoidal endothelium within the diaphysis) by immunohistochemistry (WT mice, $\mathrm{n}=27$; ITP mice, $\mathrm{n}=27$; ITP mice treated with ATRA $5 \mathrm{mg} / \mathrm{kg}$ intraperitoneal injection, $\mathrm{n}=15$; ITP mice treated with ATRA $20 \mathrm{mg} / \mathrm{kg}$ intraperitoneal injection, $\mathrm{n}=21$ ). (K) Quantification of the vascular area within marrow vessels by immunohistochemistry (WT mice, $n=27$; ITP mice, $n=27$; ITP mice treated with ATRA 5 mg/kg intraperitoneal injection, $n=15$; ITP mice treated with ATRA $20 \mathrm{mg} / \mathrm{kg}$ intraperitoneal injection, $\mathrm{n}=21)$. (I-K) Tukey tests. 
etic markers CD34 and CD45 (Figure 5E). CCK8 proliferative assays were conducted on MSC at days 1, 3, and 7 with passages 7-12. The growth curves showed a lower proliferative capacity of MSC from ITP mice (Figure 5B), while annexin $\mathrm{V}$ studies showed a higher rate of apoptosis $(P<0.001)$ (Figure 5C, D). Enhanced complement activation was confirmed by marrow immunohistochemistry, which showed increased deposition of C5b-9 (Figure 5F). The levels of IL-1 $\beta$ were significantly higher in supernatants of bone marrow from ITP mice than in those from WT mice $(P=0.000)$ (Figure $5 \mathrm{G})$, while the levels of CXCL12 were significantly lower $(P=0.011)$ (Figure 5G).

Similar to the findings in the marrow of patients from the MSC-ITP-C ${ }^{+}$group, CXCL12 fluorescence intensity was increased in the bone-associated marrow and decreased in the central marrow in ITP mice (Figure $5 \mathrm{H}$ ), implying a CXCL12 gradient toward the endosteal niche. Increased fluorescence intensity of IL-1 $\beta$ and numbers of TUNEL ${ }^{+} \mathrm{CD} 0^{+} \mathrm{MSC}$ in the central marrow were also identified in ITP mice (Online Supplementary Figures $S 4 B$ and $S 5 B)$. Notably, there was a marked vascular dilation in the area of the bone marrow occupied by the vasculature in ITP mice (Figure 5H, K). The vascular dilation was accompanied by a decrease in the megakaryocytes associated with sinusoids in ITP mice (Figure 5H-J).

ATRA $(5 \mathrm{mg} / \mathrm{kg}$ or $20 \mathrm{mg} / \mathrm{kg}$ dissolved in dimethyl sulfoxide) was administered for 10 days by intraperitoneal injection. The platelet counts in the group treated with 20 $\mathrm{mg} / \mathrm{kg}$ ATRA recovered, although they remained below the platelet counts of WT mice (Figure 5A). This group also exhibited improved MSC proliferation (Figure $5 \mathrm{~B}$ ) and less MSC apoptosis (Figure 5C, D), decreased levels of
A

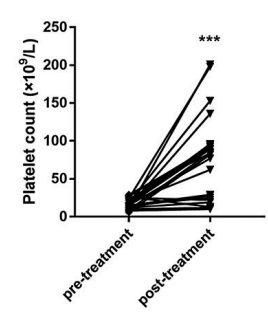

B

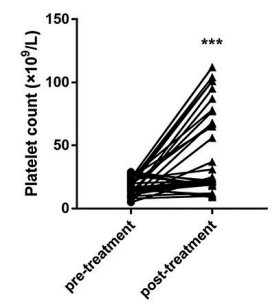

$E$

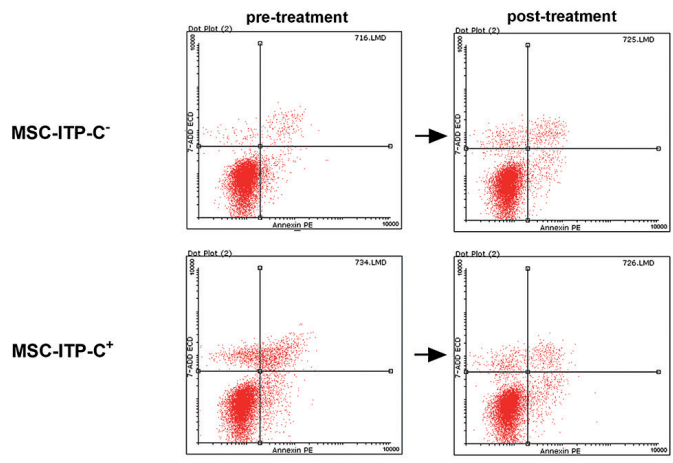

C

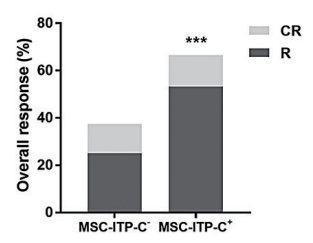

D

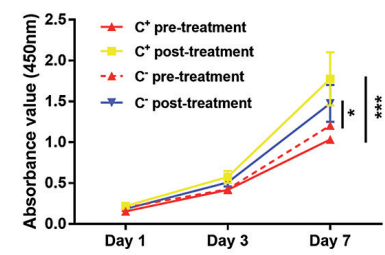

$\mathrm{F}$
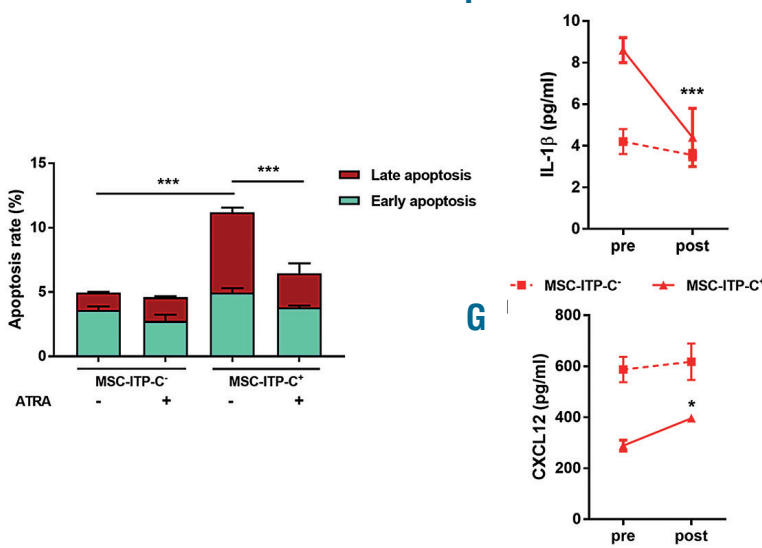

H
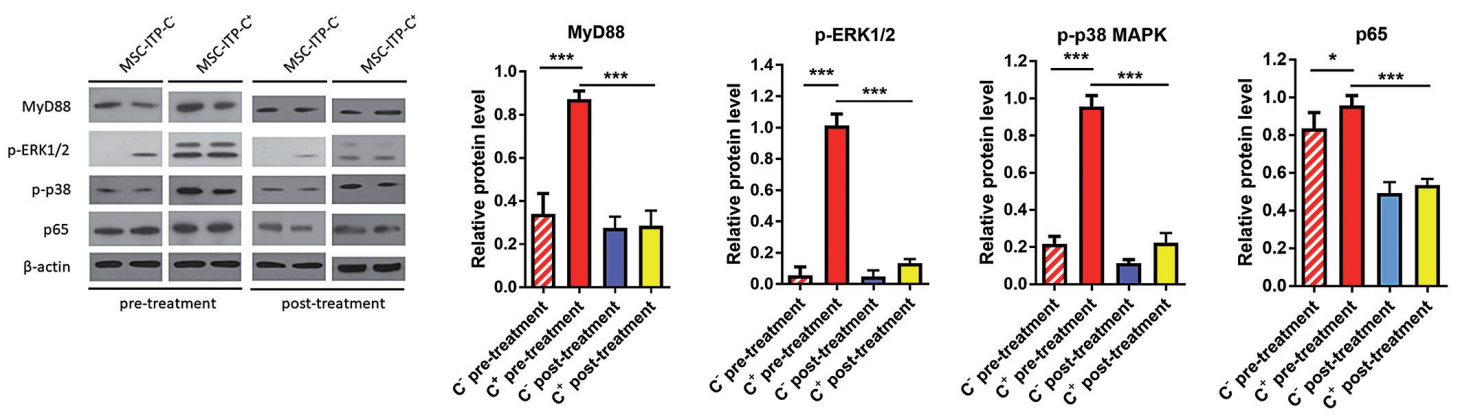

Figure 6. In vivo treatment with all-trans retinoic acid improved the function of bone marrow mesenchymal stem cells from patients with immune thrombocytopenia. (A, B) The effect of all-trans retinoic acid (ATRA) on peripheral platelet counts of the two groups of patients with immune thrombocytopenia (ITP) [MSC-ITP-C ${ }^{+}$ patients with ITP with complement deposition on their mesenchymal stem cells (MSC); MSC-ITP-C: patients with ITP without complement deposition on their MSC] MSC-ITP-C $C^{+}$group $(n=26)$ and MSC-ITP-C group $(n=32)$. Paired $t$-tests. (C) The proportions of patients with an overall response (OR) or response (R) in the MSC-ITP$\mathrm{C}^{+}$group $(\mathrm{n}=26)$ and the MSC-ITP-C group $(\mathrm{n}=32)$. Student $t$-tests. (D) The growth curves of bone marrow MSC from patients in the MSC-ITP-C $\mathrm{C}^{+}$group $(\mathrm{n}=26)$ and the MSC-ITP-C group $(n=32)$ before and after treatment with ATRA. Covariance analyses. (E) Cell apoptosis of bone marrow MSC of the patients from the MSC-ITP-C group $(n=26)$ and the MSC-ITP-C group $(n=32)$ before and after treatment with ATRA. $\chi^{2}$ tests for comparisons between pre-treatment groups. Paired $t$-tests for comparisons of samples of patients from pre- and post-treatment groups. (F, G) The levels of interleukin-1 $\beta$ (IL-1 $\beta$ ) and CXCL12 in MSC lysates (MSC-ITP-C ${ }^{+}$group, $n=26$; MSC-ITP-C group, $\mathrm{n}=32$; paired $t$-tests). (Continued on next page) 
IL-1 $\beta$ in bone marrow supernatants, as well as downregulated IL-1 $\beta$ fluorescence intensity and reduced TUNEL ${ }^{+} \mathrm{CD} 90^{+} \mathrm{MSC}$ in the central marrow (Online Supplementary Figures $S 4 D$ and $S 5 D$ ). In contrast, the group treated with $5 \mathrm{mg} / \mathrm{kg}$ ATRA did not show marked improvement of the above-mentioned parameters (Figure 5A-D, G, Online Supplementary Figures S4C and S5C).

The group treated with $20 \mathrm{mg} / \mathrm{kg}$ ATRA displayed increased CXCL12 fluorescence intensity in the central marrow and decreased intensity in the bone-associated marrow (Figure 5H), implying a reversal of the CXCL12 gradient toward the vascular niche. A reduction in vascular area and restoration of megakaryocytes associated with sinusoids in the marrow were also demonstrated in this group (Figure $5 \mathrm{H}-\mathrm{K}$ ). The group treated with $5 \mathrm{mg} / \mathrm{kg}$ did not show significant changes in vascular area or megakaryocyte occupation. These results mirrored the complement activation, CXCL12 and megakaryocyte shift toward the bone-associated marrow in ITP mice. Administration of $20 \mathrm{mg} / \mathrm{kg}$ ATRA could regulate the CXCL12 and megakaryocyte shift toward the vasculature and promote platelet production.

Clinical responses to all-trans retinoic acid in patients with immune thrombocytopenia

Patients from both the MSC-ITP-C ${ }^{+}$and MSC-ITP-C groups were given oral ATRA (10 mg twice daily) and concomitant therapy with oral danazol (200 mg twice daily). Online Supplementary Table S2 shows the baseline characteristics of the ITP patients. At the 12-week follow up, the proportion of patients with an overall response was significantly higher in the MSC-ITP-C ${ }^{+}$group (19 of 26 patients, $73.08 \%$ ) than in the MSC-ITP-C group (13 of 32 patients, $40.63 \% ; P=0.013$ ) (Figure 6C). There were no grade 3 or worse adverse events. No patients required a dose reduction because of adverse events. The main drug-related
I

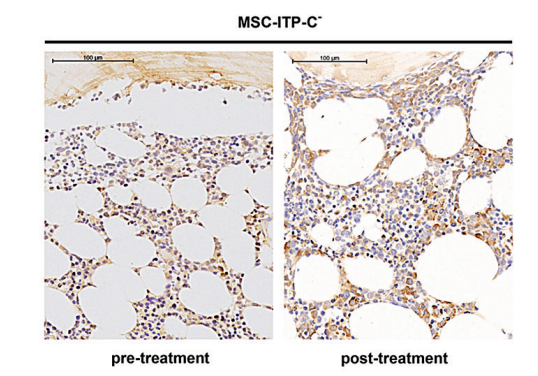

J

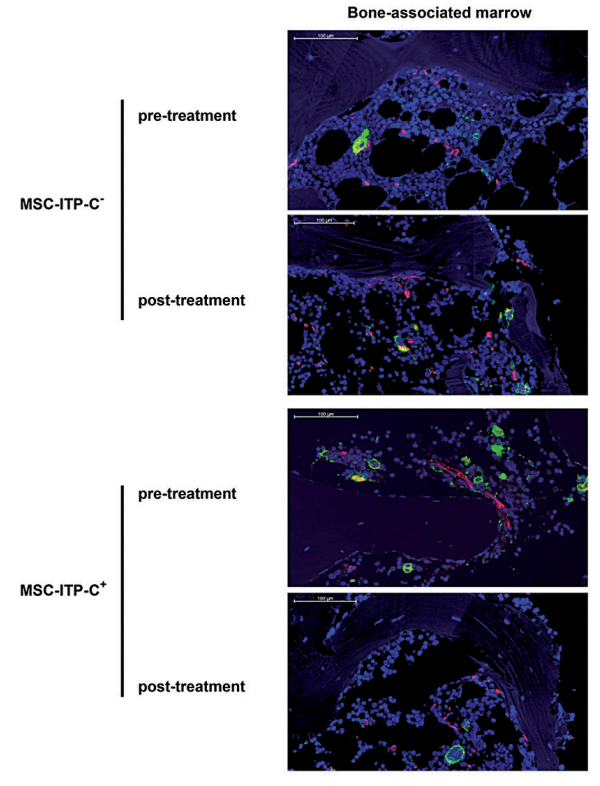

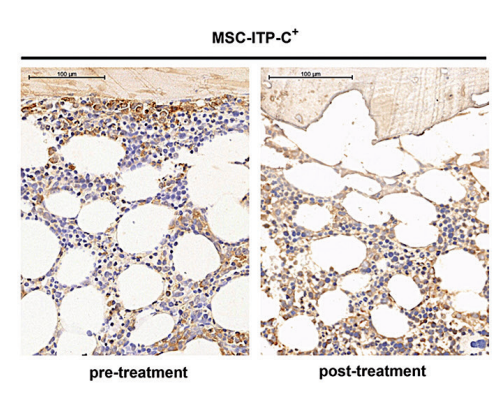

Central marrow
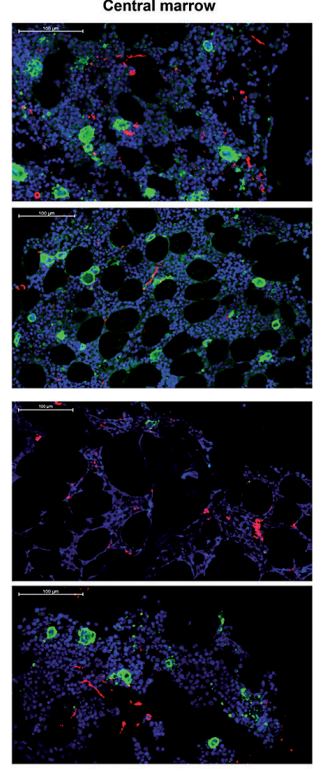

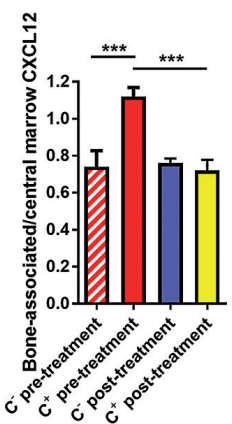

K
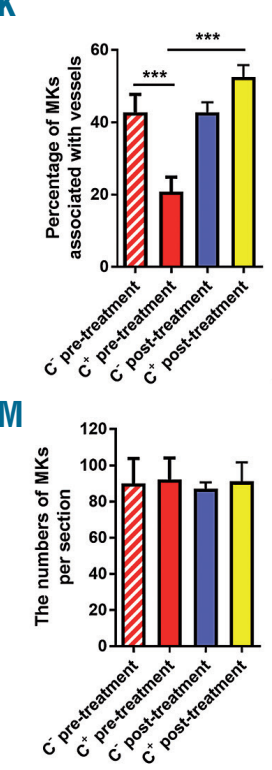

$\mathrm{L}$

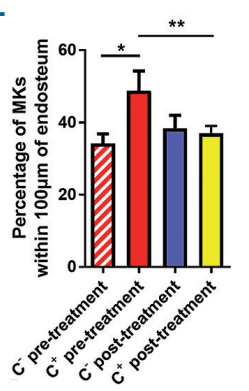

N

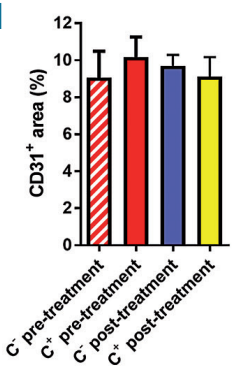

Figure 6. (Continued from previous page). (H) Phosphorylation of MyD88, ERK1/2, p38 MAPK and NF- $\mathrm{KB}$ in MSC of patients from the MSC-ITP- $\mathrm{C}^{+}$group ( $\mathrm{n}=8$ ) and the MSC-ITP-C group $(\mathrm{n}=8)$ before and after treatment with ATRA. Student $t$-tests for comparisons between pre-treatment groups. Paired $t$-tests for comparisons of samples from patients in the pre- and post-treatment groups. (I) Representative images of radioactive in situ hybridization with CXCL12 antisense probe [CXCL12 transcripts: brown pseudocolor; 4',6-diamidino-2-phenylindole (DAPI): blue] on marrow sections of the patients from the MSC-ITP-C ${ }^{+}$and the MSC-ITP-C groups. Ratio of CXCL12 transcript area in the bone-associated region (between 0-100 $\mu \mathrm{m}$ from the endosteal surface within the diaphysis) compared to an immediately adjacent region of the same size (between 100-200 $\mu \mathrm{m}$ from the endosteal surface) for ITP patients before and after ATRA treatment. (J) Representative images of bone marrow immunohistochemistry for CD41 (megakaryocytes, green) and CD31 (vascular endothelium, red) for patients from the MSC-ITP-C ${ }^{+}$and MSC-ITP-C groups. (K, L) Quantification of CD41 megakaryocytes physically associated with $\mathrm{CD} 31^{+}$vessels (between 0-10 $\mu \mathrm{m}$ from the sinusoidal endothelium within the diaphysis) and in the bone-associated region (within $100 \mu \mathrm{m}$ of the endosteal surface within the diaphysis). (M, N) CD41 megakaryocytes per section and vessel densities by immunofluorescence staining for CD31. (I, K-N) The MSC-ITP-C ${ }^{+}$group, $n=36$; the MSC-ITP-C group, $n=36$; Scale bar: $100 \mu \mathrm{m} ; \chi^{2}$ tests for comparisons between pre-treatment groups. Paired $t$-tests for comparisons of samples from patients in the pre- and post-treatment groups. 
toxic effects were skin desquamation, headache and dizziness, liver injury, edema, amenorrhea, hypertension and gastrointestinal disorders in the follow-up. Among patients who achieved an overall response, the median time to the treatment response was 34.87 days and the peak platelet count was $105.72 \times 10^{9} / \mathrm{L}^{\text {in }}$ the MSC-ITP-C ${ }^{+}$ group compared to 39.00 days and a peak platelet count of $80.62 \times 10^{9} / \mathrm{L}$ in the MSC-ITP-C group (Table 1).

To further clarify the mechanisms of the effect of ATRA in ITP, bone marrow MSC were isolated from ITP patients who had received 12 weeks of treatment. Compared with the baseline levels before ATRA therapy, the proliferative capacity, apoptosis, secretion of IL-1 $\beta$ and CXCL12, activation of IL-1R/MyD88/NF- $\mathrm{KB}$, ERK1/2 and p38 MAPK signaling pathways were altered, with trends similar to those in the in vitro studies in the patients from the MSCITP-C $C^{+}$group after treatment (Figure 6D-H). In contrast, no significant differences were found between pre-treatment and post-treatment levels in patients from the MSC-ITPC' group (Figure 6D-H).

Unsurprisingly, patients from the MSC-ITP-C+ group after treatment had overall increases in CXCL12 and megakaryocytes in the central marrow and decreases in the bone-associated marrow (Figure 6I, J), implying a CXCL12 gradient toward the sinusoids again. No significant differences of marrow CXCL12 distribution and spatial occupation of megakaryocytes were exhibited in the patients from the MSC-ITP-C group after treatment (Figure 6I, J). The numbers of megakaryocytes and the density of vessels did not differ before and after treatment (Figure 6J). These data support the hypothesis that ATRA therapy contributed to changes in the location of CXCL12 production and the altered megakaryocyte location in the bone marrow niche for platelet generation.

\section{Discussion}

Thrombopoiesis, a complex biological process initiated by pluripotent hematopoietic stem cells in the bone marrow, involves interactions with many cell types that contribute to the bone marrow niche, including osteoblasts, perivascular cells, endothelial cells, MSC, and various mature immune cells. Abnormalities during any stage of thrombopoiesis can influence platelet production. ${ }^{35}$ In the current study, we describe MSC impairment in the bone marrow of patients with ITP resulting from complementderived perturbations in the IL-1//IL-1R/NF-KB, ERK1/2 and p38 MAPK signaling pathways. Importantly, ATRA was found to decrease IL-1 $\beta$ mRNA expression and increase promoter DNA methylation. ATRA promoted functional recovery of MSC-ITP-C $\mathrm{C}^{+}$in vitro and in vivo, which facilitated the re-location of CXCL12 toward the vascular niche, enhanced megakaryocyte localization in the thrombopoietic niche and consequently promoted circulating platelet production (Figure 7).

Platelet destruction in ITP occurs through a variety of different immune-mediated mechanisms..$^{36}$ Current theories propose that antibodies against platelet-induced Fc-mediated phagocytosis, anti-GPIb/IX-mediated desialylation and the activation of cytotoxic lymphocytes are involved in the pathogenesis of ITP. ${ }^{1,37-39}$ The complement system has long been suspected to participate in platelet elimination in patients with ITP. ${ }^{1.4}$ Activation of the complement cascade was found to play a role in the pathogenesis of ITP
Table 1. Responses and outcomes of immune thrombocytopenia patients with or without complement deposition on mesenchymal stem cells.

\begin{tabular}{|c|c|c|c|}
\hline & $\begin{array}{l}\text { MSC-ITP-C } \\
(n=26)\end{array}$ & $\begin{array}{l}\text { MSC-ITP-C: } \\
(n=32)\end{array}$ & $P$ value \\
\hline \multicolumn{4}{|l|}{ Treatment response (\%) } \\
\hline Overall response & $19(73.08)$ & $13(40.63)$ & 0.013 \\
\hline Complete response & $4(15.38)$ & $4(12.50)$ & \\
\hline Response & $15(57.69)$ & $9(28.13)$ & \\
\hline No resonse & $7(26.92)$ & $19(59.37)$ & \\
\hline Blood component transfusion (\%) & $16(61.54)$ & $15(46.88)$ & 0.266 \\
\hline \multicolumn{4}{|l|}{ Significant bleeding * $(\%)$} \\
\hline $\begin{array}{l}\text { For patients with a response/ } \\
\text { complete response }\end{array}$ & $0(0)$ & $1(3.13)$ & 0.363 \\
\hline $\begin{array}{l}\text { Time to response, days, median } \\
\text { (range) }\end{array}$ & $34.89(23-52)$ & $39.00(27-53)$ & 0.190 \\
\hline $\begin{array}{l}\text { Peak platelet count, } \times 10^{9} / \mathrm{L} \text {, median } \\
\text { (range) }\end{array}$ & $105.72(62-201)$ & $80.62(37-112)$ & 0.048 \\
\hline
\end{tabular}

via complement-mediated generation of platelet microparticles in the peripheral blood. Activation of the complement system in the bone marrow of ITP patients was explored for the first time in this study. The observed enhanced deposition of C4d on MSC from patients with ITP was consistent with activation of the classical complement pathway. The lack of observed increases in C3b deposition on MSC from ITP patients indicated that the alternative complement pathway was not activated in bone marrow or that the extent of activation was at least less than the test threshold. Moreover, of 26 patients in the MSC-ITP-C ${ }^{+}$group, five $(19.2 \%)$ did not have detectable platelet antibodies, whereas of 32 patients in the MSC-ITPC group, $14(43.7 \%)$ tested positive. Based on our results, there was not a one-to-one correspondence between the presence of autoantibodies and complement activation in the niche (Online Supplementary Table S3).

MSC residing in the bone marrow have long been believed to participate in regulating the balance between hematopoietic stem cell self-renewal and differentiation. ${ }^{40}$ In addition to their self-renewal properties and potential to differentiate, MSC play crucial roles in immune modulatory functions. ${ }^{41} \mathrm{We}$ and others recently reported that MSC from ITP patients lose their conventional proliferation capacity resulting in defective immunoregulation. . $^{17,19,42,43}$ However, the mechanism underlying these abnormalities remains unknown. In the present study, we not only investigated the enhanced complement deposition on the surface of MSC from ITP patients, but also explored the underlying genetic and molecular changes that give rise to deficiencies in MSC, which were associated with activation of the complement cascade in bone marrow.

Complement components can enhance pro-inflammatory Toll-like receptor-mediated signaling in phagocytes, leading to increased production of IL- $1 \beta . .^{20-22}$ IL- $1 \beta$ is critically involved in several inflammatory diseases and its levels are elevated in many conditions characterized by complement overactivation. Furthermore, Martino et al. reported that IL-1 / /IL-1R1/MyD88 signaling impairs MSC proliferation, migration and differentiation. ${ }^{44}$ However, it is not known whether and how complement and IL- $1 \beta$ are linked and their roles in MSC remain elusive. The biology 


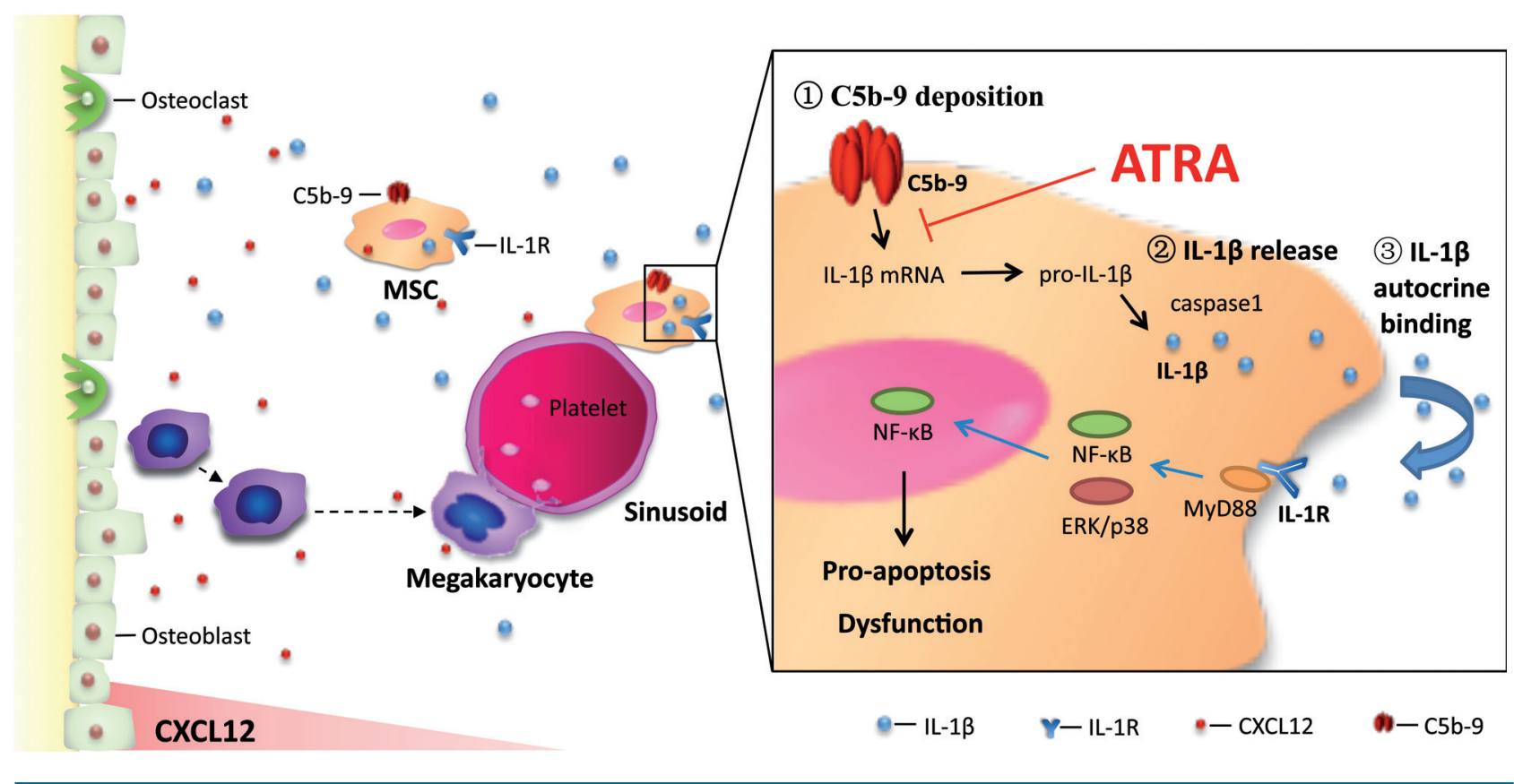

Figure 7. Proposed model for aberrantly enhanced complement activation and interleukin-1 $\beta$ expression in mesenchymal stem cells from patients with immune thrombocytopenia. C5b-9 deposition and the associated pro-inflammatory cytokine interleukin- $1 \beta$ (IL-1 $\beta$ ) could induce dysfunction of bone marrow mesenchymal stem cells (MSC) in immune thrombocytopenia (ITP), and consequently cause dynamic changes in niche CXCL12 and alterations in the location of megakaryocytes in the bone marrow. All-trans retinoic acid (ATRA) has an inhibitory effect on IL-1 $\beta$ mRNA expression and increases promoter DNA methylation, further promoting in vitro and in vivo functional recovery of MSC from ITP patients with complement deposition on their MSC (MSC-ITP-C ${ }^{+}$), which facilitates the re-location of CXCL12 towards the vascular niche, and enhances megakaryocyte localization in thrombopoietic niche.

of IL-1 $\beta$ is complex with the release of functional IL-1 $\beta$ requiring two steps. The first, rate-limiting step is the transcription of its precursor, pro-IL-1 $\beta$. The second step involves the conversion of the inactive precursor into the biologically active IL-1 $\beta$. This process is mediated by inflammasomes that activate cysteine protease caspase$1{ }^{45} \mathrm{IL}-1 \beta$ and caspase- 1 are expressed by monocytes, as previously reported, and interestingly, these molecules are also expressed by MSC. . $^{2,25}$ The microarray data from this study showed an absolute increase in mRNA expression of pro-inflammatory cytokines, including IL-1 $\beta$, in the MSC-ITP-C ${ }^{+}$group. Further results suggested a complement-IL-1 $\beta$ loop in MSC, which contributed to the dysfunction and apoptosis of MSC in ITP. Interestingly, the numbers of hematopoietic stem cells and megakaryocyte progenitors were not significantly different between the MSC-ITP-C+, MSC-ITP-C and MSC-control groups (Online Supplementary Figure S9). Whether MSC or osteoblasts (derived from MSC) are the primary target for attack by the complement-IL-1 $\beta$ loop in ITP remains to be elucidated.

Several studies have implicated chemokine CXCL12 signaling, through the CXCR4 receptor, in the maturational localization of megakaryocytes to the vascular niche. Some cell types within the bone marrow produce CXCL12, including osteoblasts, endothelial cells, and mesenchymal stromal cell populations. ${ }^{46,47}$ Despite the growing body of evidence indicating a role for CXCL12/CXCR4 in megakaryopoiesis, the effects of deficient MSC on CXCL12 distribution and megakaryocyte localization in ITP remain unknown. Here, we observed decreased expression of CXCL12 mRNA and protein in
MSC cell lysates, accompanied by an overall increase in bone-associated/central marrow CXCL12 and a decrease in the megakaryocytes associated with sinusoids in MSCITP-C+. In addition to enlarging the sample size, further investigations, such as establishing a transgenic murine model, would specifically help to identify the distribution of CXCL12 in the bone marrow niche. CXCR4 expression on megakaryocyte surfaces was higher in patients with ITP, with no difference between the MSC-ITP-C ${ }^{+}$and the MSC-ITP-C groups (Online Supplementary Figure S10).

ATRA has revolutionized the therapy of acute promyelocytic leukemia. ${ }^{26}$ ATRA also shows potential as an immune modulator, given that inflammatory cytokine production was affected in the presence of ATRA. In vitro studies have shown that natural regulatory $T$ cells treated with ATRA are resistant to T-helper cell conversion and maintain FOXP3 expression under inflammatory conditions. ATRA could also promote transforming growth factor- $\beta$-induced regulatory $T$ cells and inhibit the differentiation of T-helper-1 and T-helper-17 cells, indicating that ATRA is a novel treatment for immune-mediated diseases. ${ }^{48,49}$ In patients with ITP, treatment with ATRA restored decreased concentrations of regulatory $\mathrm{T}$ cells and IL-10, reduced FOXP3 expression and restored the balance of macrophages towards M2. ${ }^{29}$ In addition, previous studies demonstrated the ability of ATRA to inhibit IL-1 $\beta$ mRNA expression and its potential role in regulating DNA promoter methylation. Importantly, we previously reported the clinical efficacy of ATRA in patients with ITP, ${ }^{27}$ although its effect on primary ITP remains unknown. These findings led us to explore whether the mechanisms of action of ATRA in the treatment of ITP are 
associated with MSC function via the regulation of IL-1 $\beta$ synthesis. Expectedly, ATRA inhibited IL-1 $\beta$ mRNA expression in MSC via upregulation of promoter DNA methylation. Further studies are warranted to confirm these data in a larger group of patients with primary ITP.

In conclusion, we have shown that enhanced complement activation and the associated pro-inflammatory cytokine IL-1 $\beta$ could induce dysfunction of bone marrow MSC in ITP and consequently cause dynamic changes in niche CXCL12 and alterations in the location of megakaryocytes in the bone marrow. ATRA is a promising therapeutic approach for repairing MSC dysfunction in primary ITP patients. The present study sheds light on complement activation in the pathogenesis of ITP and provides new shreds of evidence confirming the general applicability of ATRA in the treatment of ITP.

\section{Acknowledgments}

This work was supported by the National Natural Science Foundation of China (n. 81470343 and n. 81670116), a Key Program of the National Natural Science Foundation of China (n. 81730004), the Beijing Natural Science Foundation (n. 7171013), the Beijing Municipal Science and Technology Commission (n. Z171100001017084), the National Key Research and Development Program of China (n. 2017YFA0105500 and n. 2017YFA0105503) and the Foundation for Innovation Research Groups of the National Natural Science Foundation of China (n. 81621001).

\section{References}

1. Toltl LJ, Nazi I, Jafari R, Arnold DM. Piecing together the humoral and cellular mechanisms of immune thrombocytopenia. Semin Thromb Hemost. 2011;37(6):631-639.

2. Najaoui A, Bakchoul T, Stoy J, et al. Autoantibody-mediated complement activation on platelets is a common finding in patients with immune thrombocytopenic purpura (ITP). Eur J Haematol. 2012;88(2): 167-174.

3. Peerschke EI, Andemariam B, Yin W, Bussel JB. Complement activation on platelets correlates with a decrease in circulating immature platelets in patients with immune thrombocytopenic purpura. Br J Haematol. 2010;148 (4):638-645

4. Zhu X, Zhang J, Wang Q, et al. Diminished expression of beta2-GPI is associated with a reduced ability to mitigate complement activation in anti-GPIIb/IIla-mediated immune thrombocytopenia. Ann Hematol. 2018;97 (4):641-654.

5. Carroll MC. The complement system in regulation of adaptive immunity. Nat Immunol. 2004;5(10):981-986.

6. Hardy RR, Hayakawa K. B cell development pathways. Annu Rev Immunol. 2001;19: 595-621.

7. Heeger PS, Kemper C. Novel roles of complement in $\mathrm{T}$ effector cell regulation. Immunobiology. 2012;217(2):216-224.

8. Kemper C, Atkinson JP. T-cell regulation: with complements from innate immunity. Nat Rev Immunol. 2007;7(1):9-18.

9. Longhi MP, Harris CL, Morgan BP, Gallimore A. Holding T cells in check--a new role for complement regulators? Trends Immunol. 2006;27(2):102-108.

10. Kemper C, Chan AC, Green JM, Brett KA, Murphy KM, Atkinson JP. Activation of human CD4+ cells with CD3 and CD46 induces a T-regulatory cell 1 phenotype. Nature. 2003;421(6921):388-392.

11. Audia S, Mahevas M, Samson M, Godeau B, Bonnotte B. Pathogenesis of immune thrombocytopenia. Autoimmun Rev. 2017;16(6): 620-632.

12. Song Y, Wang YT, Huang XJ, Kong Y. Abnormalities of the bone marrow immune microenvironment in patients with immune thrombocytopenia. Ann Hematol. 2016;95 (6):959-965.

13. Zufferey A, Kapur R, Semple JW. Pathogenesis and therapeutic mechanisms in immune thrombocytopenia (ITP). J Clin Med. 2017;6(2):pii:E16.
14. Ji L, Zhan Y, Hua F, et al. The ratio of Treg/Th17 cells correlates with the disease activity of primary immune thrombocytopenia. PLoS One. 2012;7(12):e50909.

15. Glennie S, Soeiro I, Dyson PJ, Lam EW, Dazzi F. Bone marrow mesenchymal stem cells induce division arrest anergy of activated T cells. Blood. 2005;105(7):2821-2827.

16. Tabera S, Pérez-Simón JA, Díez-Campelo $M$, et al. The effect of mesenchymal stem cells on the viability, proliferation and differentiation of B-lymphocytes. Haematologica. 2008;93(9):1301-1309.

17. Zhang JM, Feng FE, Wang $\mathrm{OM}$, et al. Platelet-derived growth factor-BB protects mesenchymal stem cells (MSCs) derived from immune thrombocytopenia patients against apoptosis and senescence and maintains MSC-mediated immunosuppression. Stem Cells Transl Med. 2016;5(12):16311643

18. He Y, Xu LL, Feng FE, et al. Mesenchymal stem cell deficiency influences megakaryocytopoiesis through the TNFAIP3/NFkappaB/SMAD pathway in patients with immune thrombocytopenia. Br J Haematol. 2018;180(3):395-411.

19. Xu LL, Fu HX, Zhang JM, et al. Impaired function of bone marrow mesenchymal stem cells from immune thrombocytopenia patients in inducing regulatory dendritic cell differentiation through the Notch-1/Jagged1 signaling pathway. Stem Cells Dev. 2017:26(22):1648-1661.

20. Laudisi F, Spreafico R, Evrard M, et al. Cutting edge: the NLRP3 inflammasome links complement-mediated inflammation and IL-1beta release. J Immunol. 2013;191(3):1006-1010.

21. Fang C, Zhang X, Miwa T, Song WC. Complement promotes the development of inflammatory T-helper 17 cells through synergistic interaction with Toll-like receptor signaling and interleukin-6 production. Blood. 2009;114(5):1005-1015.

22. Zhang X, Kimura Y, Fang C, et al. Regulation of Toll-like receptor-mediated inflammatory response by complement in vivo. Blood. 2007;110(1):228-236

23. Hua F, Li Y, Zhao X, et al. The expression profile of toll-like receptor signaling molecules in CD19(+) B cells from patients with primary immune thrombocytopenia. Immunol Lett. 2016;176:28-35.

24. Liu N, Wang H, Han G, Tian J, Hu W, Zhang J. Alleviation of apoptosis of bone marrowderived mesenchymal stem cells in the acute injured kidney by heme oxygenase-1 gene modification. Int J Biochem Cell Biol. 2015;69:85-94.

25. Romieu-Mourez R, Francois M, Boivin MN Bouchentouf M, Spaner DE, Galipeau J. Cytokine modulation of TLR expression and activation in mesenchymal stromal cells leads to a proinflammatory phenotype. J Immunol. 2009;182(12):7963-7973.

26. Cicconi L, Lo-Coco F. Current management of newly diagnosed acute promyelocytic leukemia. Ann Oncol. 2016;27(8):14741481.

27. Feng FE, Feng R, Wang $M$, et al. Oral alltrans retinoic acid plus danazol versus danazol as second-line treatment in adults with primary immune thrombocytopenia: a multicentre, randomised, open-label, phase 2 trial. Lancet Haematol. 2017;4(10):e487e496.

28. Panzer S, Pabinger I. Is oral all-trans retinoic acid plus danazol a refinement of secondline therapy for primary immune thrombocytopenia in adults? Lancet Haematol. 2017;4(10):e457-e458.

29. Feng $\mathrm{Q}, \mathrm{Xu} M$, Yu YY, et al. High-dose dexamethasone or all-trans-retinoic acid restores the balance of macrophages towards M2 in immune thrombocytopenia. J Thromb Haemost. 2017;15(9):1845-1858.

30. Provan D, Stasi R, Newland AC, et al. International consensus report on the investigation and management of primary immune thrombocytopenia. Blood. 2010; 115(2):168-186.

31. Neunert C, Lim W, Crowther M, Cohen A, Solberg LJ, Crowther MA. The American Society of Hematology 2011 evidence-based practice guideline for immune thrombocytopenia. Blood. 2011;117(16):4190-4207.

32. Laguri C, Arenzana-Seisdedos F, LortatJacob H. Relationships between glycosaminoglycan and receptor binding sites in chemokines-the CXCL12 example. Carbohydr Res. 2008;343(11):2018-2023.

33. Niswander LM, Fegan KH, Kingsley PD, McGrath KE, Palis J. SDF-1 dynamically mediates megakaryocyte niche occupancy and thrombopoiesis at steady state and following radiation injury. Blood. 2014;124(2): 277-286.

34. Chow L, Aslam R, Speck ER, et al. A murine model of severe immune thrombocytopenia is induced by antibody- and CD8+ T cellmediated responses that are differentially sensitive to therapy. Blood. 2010;115(6): 1247-1253.

35. Wang L, Li Y, Hou M. Idiopathic thrombocytopenic purpura and dysmegakaryocy- 
ATRA protects impaired BM MSC in ITP

topoiesis. Crit Rev Oncol Hematol. 2007;64(2):83-89.

36. Cine DB, Bussed JB, Liebman HA, Lining PE. The ITP syndrome: pathogenic and lincal diversity. Blood. 2009;113(26):65116521.

37. Chang F, Chr X, Wang L, et al. Cell-mediated lysis of tautologous platelets in chronic idiopathic thrombocytopenic purpura. Eur J Haematol. 2006;76(5):427-431.

38. Li J, van der Wal DE, Zhu G, et al. Desialylation is a mechanism of Fc-independent platelet clearance and a therapeutic target in immune thrombocytopenia. Nat Commune. 2015;6:7737.

39. Zhao C, Li X, Chang F, Wang L, Peng J, How $M$. Increased cytotoxic T-lymphocyte-mediate cytotoxicity predominant in patients with idiopathic thrombocytopenic purpura without platelet autoantibodies. Haematological. 2008;93(9):1428-1430.

40. Almeida-Porada G, Prada CD, Tran N, Zanjani ED. Cotransplantation of human stromal cell progenitors into preimmune fetal sheep results in early appearance of human donor cells in circulation and boosts cell levels in bone marrow at later time points after transplantation. Blood. 2000;95 (11):3620-3627.

41. English K, Ryan JM, Tobin L, Murphy MJ, Barry FP, Mahon BP. Cell contact, prostaglandin $\mathrm{E}(2)$ and transforming growth factor beta 1 play non-redundant roles in human mesenchymal stem cell induction of CD4+CD25(High) forkhead box P3+ regulatory $\mathrm{T}$ cells. Chin Exp Immunol. 2009;156(1):149-160.

42. Pérez-Simón JA, Tabera S, Sarasquete ME, et al. Mesenchymal stem cells are functionally abnormal in patients with immune throwbocytopenic purpura. Cytotherapy. 2009;11(6):698-705.

43. Chang D, Li H, Ma L, et al. The defective bone marrow-derived mesenchymal stem cells in patients with chronic immune thrombocytopenia. Autoimmunity. 2014;47 (8):519-529.

44. Martino MM, Maruyama K, Kuhn GA, et al. Inhibition of IL-1R1/MyD88 signalling promotes mesenchymal stem cell-driven tissue regeneration. Nat Commun. 2016;7:11051.

45. de Mooij C, Netea MG, van der Velden W, Blijlevens N. Targeting the interleukin-1 pathway in patients with hematological disorders. Blood. 2017;129(24):3155-3164.

46. Wynn RF, Hart CA, Corradi-Perini C, et al. A small proportion of mesenchymal stem cells strongly expresses functionally active CXCR4 receptor capable of promoting migration to bone marrow. Blood. 2004;104 (9):2643-2645.

47. Ding L, Morrison SJ. Haematopoietic stem cells and early lymphoid progenitors occupy distinct bone marrow niches. Nature. 2013;495(7440):231-235.

48. Lu ZM, Wang KP, Ma J, Gus ZS. The role of all-trans retinoic acid in the biology of Foxp3+ regulatory $\mathrm{T}$ cells. Cell $\mathrm{Mol}$ Immunol. 2015;12(5):553-557.

49. Lu L, Lan Q, Li Z, et al. Critical role of alltrans retinoic acid in stabilizing human natural regulatory $\mathrm{T}$ cells under inflammatory conditions. Proc Natl Aced Sci U S A. 2014;111(33): E3432-E3440.

haematological | 2019; 104(8)

1675 